\title{
Exposure to negative socio-emotional events induces sustained alteration of resting-state brain networks in the elderly
}

Sebastian Baez Lugo ( $\nabla$ sebastian.baezlugo@unige.ch )

University of Geneva https://orcid.org/0000-0002-7781-2387

Yacila Deza-Araujo

University Of Geneva https://orcid.org/0000-0002-2624-077X

Fabienne Collette

University of Liège https://orcid.org/0000-0001-9288-9756

Patrik Vuilleumier

Lab NIC https://orcid.org/0000-0002-8198-9214

Olga Klimecki

University of Geneva https://orcid.org/0000-0003-0757-7761

and The Medit-Ageing Research group

\section{Research Article}

Keywords: Ageing, Anxiety, Rumination, Emotional resilience, Default Mode Network, Functional connectivity, Amygdala, Insula, Posterior cingulate cortex, fMRI

Posted Date: November 3rd, 2020

DOI: https://doi.org/10.21203/rs.3.rs-91196/v2

License: (c) (i) This work is licensed under a Creative Commons Attribution 4.0 International License.

Read Full License 
1 Title:

2 Exposure to negative socio-emotional events induces sustained alteration of

3 resting-state brain networks in the elderly

4

5

6 Authors:

7 Sebastian Baez Lugo ${ }^{1,2, *}$, Yacila I. Deza-Araujo ${ }^{1,2}$, Fabienne Collette ${ }^{3}$, Patrik Vuilleumier ${ }^{1,2}$, Olga

8 Klimecki ${ }^{1,4}$, and the Medit-Ageing Research Group

9

10 Affiliations:

$11{ }^{1}$ Swiss Center for Affective Sciences, University of Geneva, Geneva, Switzerland.

$12{ }^{2}$ Laboratory for Behavioral Neurology and Imaging of Cognition, Department of Neuroscience,

13 Medical School, University of Geneva, Geneva, Switzerland.

$14{ }^{3}$ GIGA-CRC In Vivo Imaging Research Unit, University of Liège, Liège, Belgium.

$15{ }^{4}$ Psychology Department, Technische Universität Dresden, Dresden, Germany.

16

$17 *$ Correspondence concerning this article should be addressed to: sebastian.baezlugo@unige.ch 18 
Abstract:

20 Socio-emotional functions seem well-preserved in the elderly. However, the long-lasting effects 21 that the exposure to others' distress may provoke in the brain remain unknown in this population. 22 To evaluate how the aging brain reacts during and after emotionally challenging social events, we 23 designed a new "task-rest" paradigm in which elderly participants $(N=127)$ underwent functional 24 magnetic resonance imaging (fMRI) while exposed to socio-emotional videos. We unveil neural 25 markers of "emotional inertia" in brain activity and connectivity following negative scenes. 26 Exposure to others' suffering induced differential activations that lingered over time into the 27 subsequent resting-state in regions of the default mode network (DMN). Moreover, emotional 28 elicitation potentiated subsequent resting-state connectivity between posterior DMN and amygdala, 29 which in turn was related to anxiety, rumination, and negative thoughts. These findings uncover 30 brain mechanisms underlying emotional resilience and empathy in the elderly and may help 31 understand how poor social stress regulation promotes neurodegenerative diseases.

32

\section{Keywords:}

34 Ageing, Anxiety, Rumination, Emotional resilience, Default Mode Network, Functional 35 connectivity, Amygdala, Insula, Posterior cingulate cortex, fMRI. 
Aging is a multifaceted process associated with many changes in bodily and mental health. While there is a general decline in physical performances and cognitive abilities in aging ${ }^{1}$, emotional functions appear to be maintained or even enhanced in older adults relative to younger adults ${ }^{2-4}$. Indeed, the elderly tend to regulate their emotional states well, a crucial capacity for affective wellbeing and healthy aging 5 . Unlike younger adults, they often prioritize social and emotional interactions over other goals ${ }^{6}$ and show a "positivity bias" in emotion perception ${ }^{7}$. In contrast, maladaptive emotional reactivity and impaired emotion regulation are related to affective psychopathologies such as anxiety, depression, worry, and rumination throughout the lifespan ${ }^{8,9}$, including in aging ${ }^{10}$. There is also growing evidence that maladaptive affective styles may represent a significant risk factor for dementia ${ }^{11-14}$, one of the primary mental health burdens in the elderly population ${ }^{15}$. However, the neural substrates underpinning proficient socio-affective processing and emotional resilience in the elderly remain unresolved and still scarcely investigated.

An important marker of maladaptive affective style is "emotional inertia", which denotes the degree to which emotions carry over from one moment to the next ${ }^{16}$. Emotional inertia may reflect unsuccessful recovery mechanisms following the offset of affective events and low resilience to stress, associated with higher risks of depression ${ }^{17,18}$ and higher trait anxiety and rumination tendencies ${ }^{19}$. Most studies of emotional inertia employed behavioral measures based on experience sampling methods ${ }^{16,20}$, e.g., requiring participants to report their affective state at different time points and measuring autocorrelations between successive time-points or events 16,21,22. More recently, a few neuroimaging studies investigated emotional inertia at the brain level using "taskrest" paradigms ${ }^{23-29}$, in which brain activity during rest at time $\mathrm{T}$ is probed as a function of different task-induced activations at time T- ${ }^{30}$. For example, positive or negative emotions evoked by images or videos were found to induce carryover effects on brain activity and/or connectivity during subsequent resting-state in default mode and affective networks ${ }^{24,28}$. These carryover effects have been observed at different time scales ranging from a few seconds ${ }^{31}$ to several minutes ${ }^{27}$, following different task instructions ranging from passive viewing through to active regulation of emotions ${ }^{23}$, and across different conditions of emotional valence and intensity ${ }^{25,26}$.

At the neural level, most brain imaging studies found carryover effects of emotions on the functional dynamics of the default mode network (DMN) either in the form of increased ${ }^{26,31}$ or decreased ${ }^{24,25}$ activity patterns in regions comprising the medial prefrontal cortex (MPFC), 
are usually active when individuals are free to let their mind wander in undisturbed conditions ${ }^{32,33}$. Similar effects have also been observed in the insula and amygdala ${ }^{24}$; two regions critically involved in emotional and social processing ${ }^{34-36}$. For instance, a slow recovery of amygdala activity (i.e., longer return to baseline level) after negative images was reported in individuals with higher neuroticism ${ }^{37}$. Slower recovery of amygdala activity after emotional videos was furthermore associated with higher anxiety traits and ruminations ${ }^{19}$. Subcortical limbic regions such as the amygdala and striatum also display sustained changes in their functional connectivity with cortical areas in medial PFC and PCC during rest after negative emotions ${ }^{24}$ and reward ${ }^{28}$. These findings converge with other studies showing that disturbances in functional connectivity of the amygdala with medial parts of the DMN at rest are associated with anxiety (e.g., decreased connectivity with $\mathrm{MPFC}^{38}$ ) and mood disorders (e.g., increased connectivity with $\mathrm{PCC}^{39}$ ). Taken together, these data suggest that long-lasting carryover effects of emotions on activity and connectivity of limbic networks may provide an important neural marker of emotional regulation style and affective resilience.

Yet, all previous neuroimaging studies of emotional carryover focused on young healthy participants. It remains unknown whether emotional inertia also occurs in the elderly population, how it is modified given the well-known "positivity bias" observed in the elderly ${ }^{2,3}$, and which brain networks are involved. Here we therefore used a similar task-rest paradigm to probe for carryover effects in a large sample of healthy elderly participants, and identify neural substrates of individual differences in emotional inertia in this population. Defining valid markers of adaptive emotion regulation abilities in a naturalistic paradigm, without making high cognitive demands required by more voluntary/explicit regulation strategies ${ }^{40}$, would be valuable to better understand affective resilience mechanisms and better predict affective risk factors associated with pathological aging and dementia ${ }^{14}$.

In addition, previous work did not assess whether emotional inertia is modulated by individual differences in empathy, which may strongly influence how people react to negative socio-affective stimuli presented in neuroimaging studies ${ }^{19,24}$, and thus how they recover from induced emotions ${ }^{27}$. Because social competences and affective empathy are relatively preserved in the elderly ${ }^{4}$, socially significant emotional events offer an optimal window to probe emotional reactivity and regulation in this population. Moreover, there is only scarce research on empathy in older people ${ }^{4,41-43}$. Behaviorally, cognitive empathy declines in older compared to younger people, while affective empathy may remain intact or even improve ${ }^{4}$. At the brain level, hemodynamic responses to the perception of others' pain were reported to be reduced in anterior insula (AI) and cingulate cortex (ACC) ${ }^{41}$; two regions implicated in pain processing, negative affect, and salience 
detection ${ }^{35,44}$. However, despite the importance of social interactions and emotional resilience for healthy aging ${ }^{45-47}$, the neural underpinnings of (mal)adaptive affective reactivity as well as their link with individual empathic skills and personality traits have not been investigated during aging yet.

To address these issues, we designed a novel "task-rest" paradigm combining two lines of research: short (10-18s) empathy inducing videos from the Socio-affective Video Task (SoVT) ${ }^{48}$ were shown interspersed with rest periods of 90 seconds (similar to Eryilmaz and colleagues ${ }^{24}$ ) while participants underwent functional magnetic resonance imaging (fMRI) of brain activity. The SoVT videos consisted of short silent scenes depicting suffering people (high emotion videos) or people in everyday life situations (low emotion videos). By adding short resting state periods after blocks of videos of each kind, the SoVT-Rest allowed us to evaluate how the aging brain reacts both during and after exposure to emotionally challenging social information. In addition, we assessed whether emotional inertia in brain networks is modulated by empathic traits and anxiety, and how it relates to rumination tendencies. We hypothesized that exposure to others' suffering (relative to neutral social situations) would (1) engage brain regions implicated in emotional saliency and empathy (i.e., insula, aMCC), (2) induce subsequent carryover effects in functional connectivity at rest between these regions and the DMN, and (3) unveil neural markers of maladaptive emotional recovery that should be associated with anxiety, ruminative thinking, and negative emotions.

\section{Material and Methods}

\section{Participants}

A total of 135 healthy elderly participants with corrected-to-normal vision and no history of neurological or psychiatric disorders, aged between 65 and 83 years, took part in our study. This session was part of the baseline visit of the Age-Well randomized clinical trial within the MeditAgeing Project ${ }^{49}$. Detailed inclusion criteria of the Age-Well randomized clinical trial are provided in Supplementary Table 1. Participants were recruited via advertising in media outlets, social media, and flyers distributed in relevant local events and places. A total of 8 participants were excluded from final data analysis due to the following a priori exclusion criteria: abnormal brain morphology $(n=3)$, extreme head motion $(n=3)$, and presence of artifacts in brain images $(n=2)$. The final sample for the current study included 127 participants $(M$ age $=68.8$ years, $S D=3.63,79$ females.

See Table 1 for participants' characteristics). This sample size was sensitive enough to detect smallto-medium-sized effects at a voxel level $(\alpha=0.001, d z=0.37)$, given a power of $1-\beta=0.8^{50}$. All 
139 participants provided written informed consent prior to participation. The Age-Well randomized

140 clinical trial was approved by the ethics committee (Comité de Protection des Personnes Nord-

141 Ouest III, Caen, France; trial registration number: EudraCT: 2016-002441-36; IDRCB: 2016-

142 A01767-44; ClinicalTrials.gov Identifier: NCT02977819).

143

\section{Questionnaires}

145 In order to account for inter-individual differences in psycho-emotional profile, participants filled

146 in different questionnaires assessing personality traits and cognitive functions, including empathy

147 (Interpersonal Reactivity Index, IRI ${ }^{51}$ ), depression (Geriatric Depression Score, GDS ${ }^{52}$ ), anxiety

148 (STAI-trait Anxiety Index, STAI ${ }^{53}$ ), emotion regulation capacities (Emotion Regulation

149 Questionnaire, ERQ ${ }^{54}$ ), and rumination levels (Rumination Response Scale, RRS ${ }^{55}$ ). The summary

150 of these questionnaires is provided in Table 1. All scores were in the normative range. For a full list

151 of tasks and measures used in the Age-Well trial, please refer to Poisnel and colleagues ${ }^{49}$. 


\begin{tabular}{|c|c|c|c|c|c|c|}
\hline \multicolumn{7}{|c|}{ Table 1. Participant characteristics } \\
\hline & & $\mathbf{n}$ & Min & Max & Mean & $\begin{array}{l}\text { Std. } \\
\text { Dev }\end{array}$ \\
\hline \multicolumn{7}{|l|}{ Demographics } \\
\hline \multirow[t]{2}{*}{ Sex } & Female & 79 & & & & \\
\hline & Male & 48 & & & & \\
\hline Age & & 127 & 65 & 83 & 68.81 & 3.63 \\
\hline Education (n. of years) & & 127 & 7 & 22 & 13.21 & 3.1 \\
\hline \multicolumn{7}{|l|}{$\begin{array}{l}\text { Personality traits and Cognitive } \\
\text { functions }\end{array}$} \\
\hline STAI & Trait & 127 & 20 & 54 & 34.57 & 7.12 \\
\hline \multirow[t]{3}{*}{ Rumination Response Scale } & Total & 126 & 22 & 68 & 35.67 & 8.55 \\
\hline & Reflection & 126 & 5 & 17 & 8.93 & 3.23 \\
\hline & Brooding & 126 & 5 & 16 & 8.06 & 2.28 \\
\hline \multirow[t]{4}{*}{ Interpersonal Reactivity Index } & Distress & 127 & 0 & 26 & 10.18 & 5.27 \\
\hline & Empathic Concern & 127 & 8 & 28 & 19.76 & 4.18 \\
\hline & Perspective Taking & 127 & 9 & 28 & 17.50 & 3.56 \\
\hline & Fantasy & 127 & 1 & 28 & 14.35 & 4.75 \\
\hline \multirow[t]{2}{*}{ Emotion regulation abilities } & Reappraisal & 127 & 6 & 40 & 29.61 & 5.79 \\
\hline & Suppresion & 127 & 4 & 28 & 16.54 & 5.19 \\
\hline Geriatric Depression Scale & Global & 127 & 0 & 11 & 1.31 & 1.77 \\
\hline
\end{tabular}

\section{Socio-affective Video Task-Rest (SoVT-Rest)}

159 The emotion-elicitation task used in this study is adapted from the previously validated Socio-

160 affective Video Task (SoVT) ${ }^{48,56}$. The SoVT aims at assessing social emotions (e.g., empathy) in 161 response to short silent videos (10-18s). During this task, participants watch 12 High Emotion (HE)

162 and 12 Low Emotion (LE) video-clips grouped in blocks of three. HE videos depict suffering people

163 (e.g., due to injuries or natural disasters), while LE videos depict people during everyday activities

164 (e.g., walking or talking). In this study, each block was followed by a resting state period of 90

165 seconds in order to assess the carryover effects of emotion elicitation on subsequent resting-state

166 brain activity (similar to Eryilmaz and colleagues ${ }^{24}$ ). This combination of both paradigms (task and 
167 rest) was specifically designed to test for emotional inertia and its relation to empathy. The combined task (called SoVT-Rest) is illustrated in Fig. 1.

Overall, three sets (V1, V2, and V3) of 24 videos each were created and randomized across participants. In the final sample, $n=42$ participants thus saw the video set V1, $n=40$ video set V2,

171 and $n=45$ video set V3. During the SoVT-Rest, these videos were presented in two separate runs,

172 with each run followed by a thought probe to assess current mental content during the last rest

173 period (after LE videos in one run and after HE videos in the other run). The order in which runs

174 were presented was randomized so that half of the participants started the experiment with a HE

175 block and the other half with an LE block. The total duration of the SoVT-Rest fMRI paradigm was

176 approximately 21 minutes, consisting of $9.5 \mathrm{~min}$ for each run plus 1 minute on average for each

177 thought probe.

178 After the fMRI session, participants watched all video clips again on a computer outside the 179 scanner and provided ratings on their subjective experience of empathy ("To what degree did you 180 feel the emotions of the characters?") as well as their subjective positive affect ("Indicate the 181 intensity of your positive emotions") and negative affect state ("Indicate the intensity of your 182 negative emotions") (translated from French), for each of the 24 videos. Each scale offered 21

183 possible responses ranging from 0 ("Not at all") to 10 ("Extremely") with increments of 0.5. The 184 order of questions was always the same: empathy, positive affect, and negative affect. The total 185 time for the out-of-scanner rating session was, on average, 10 minutes. Onset times and response 186 times for both neuroimaging and behavioral tasks were collected via the Cogent toolbox (developed 187 by Cogent 2000 and Cogent Graphics) implemented in Matlab 2012 (Mathworks Inc., Natick, MA, 188 USA). 


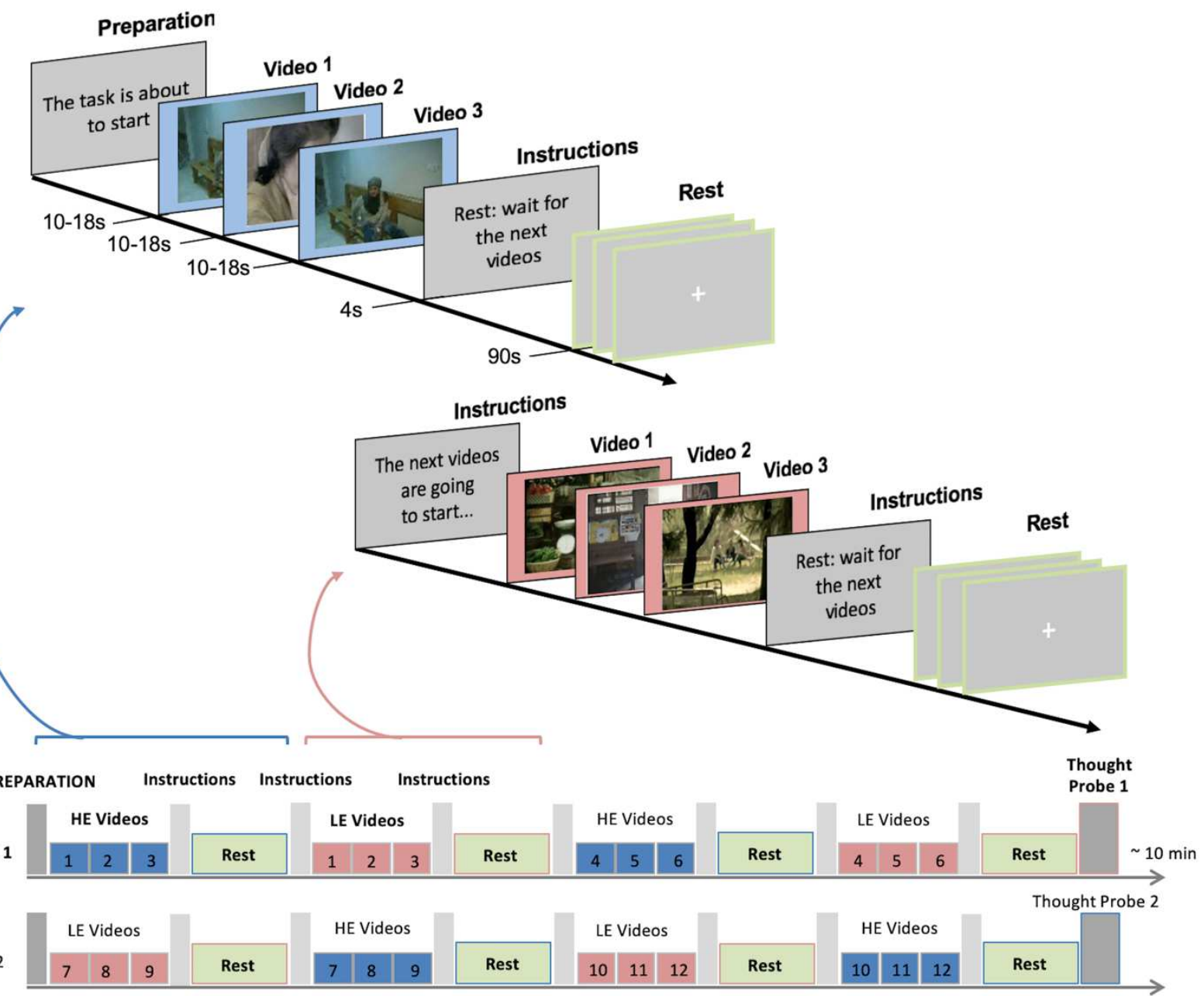

Figure 1. Experimental design: (a) SoVT-Rest paradigm: 12 High Emotion (HE) and 12 Low Emotion (LE) videos were presented grouped in blocks of three. HE videos depict suffering people (e.g., due to injuries or natural disasters), while LE videos depict people during everyday activities (e.g., walking or talking). Each block of three videos is followed by a resting state period of 90 seconds. (b) Each run ends with a thought probe in which participants verbally express what they had been thinking and/or feeling during the last rest period (via a microphone), once following a LE block and once following a HE block. The order of the runs was randomized between participants.

\section{Behavioral data analysis.}

201 We performed a repeated measures multivariate analysis of variance (MANOVA, with Pillai's trace 202 statistics) with the within-subject factor "video type" (HE and LE), the between-subject factor 203 "video set" (V1, V2, V3), and three dependent variables: ratings of empathy, positive affect, and 204 negative affect. This was followed up by pairwise $t$-tests. We also computed Spearman's rank 205 correlations between these different scores. Additionally, we performed correlation analyses 206 between the ratings of empathy, positive affect, and negative affect of videos and the relevant 207 personality traits measured by the above-mentioned questionnaires, using non-parametric 
Spearman's rank correlations because some of these variables were not normally distributed. All statistical analyses are reported with a significance level of $p<0.05$, and when necessary, $p$-values are corrected for multiple comparisons using the False Discovery Rate (FDR) method ${ }^{57}$. The statistical analyses were performed with R studio (version 3.6.1) and the corresponding graphs were created with ggplot2 (version 3.2.1).

\section{Acquisition and preprocessing of MRI data.}

Magnetic Resonance Imaging (MRI) scans were acquired at the GIP Cyceron (Caen, France) using a Philips Achieva (Eindhoven, The Netherlands) 3T scanner with a 32-channel head coil. Participants were provided with earplugs to protect hearing and their heads were stabilized with foam pads to minimize head motion. A high-resolution T1-weighted anatomical volume was first acquired using a 3D fast field echo sequence (3D-T1-FFE sagittal; repetition time $=7.1 \mathrm{~ms}$; echo time $=3.3 \mathrm{~ms}$; flip angle $=6^{\circ} ; 180$ slices with no gap; slice thickness $=1 \mathrm{~mm}$; field of view $=$ $256 \times 256 \mathrm{~mm}^{2}$; in plane resolution $=1 \times 1 \mathrm{~mm}^{2}$ ). Blood oxygen level-dependent (BOLD) images were acquired during the SoVT-Rest task with a T2*-weighted asymmetric spin-echo echo-planar sequence (each run $\sim 10.5 \mathrm{~min}$; $\mathrm{TR}=2000 \mathrm{~ms}, \mathrm{TE}=30 \mathrm{~ms}$, flip angle $=85^{\circ}, \mathrm{FOV}=240 \times 240$ $\mathrm{mm}^{2}$, matrix size $=80 \times 68 \times 33$, voxel size $=3 \times 3 \times 3 \mathrm{~mm}^{3}$, slice gap $=0.6 \mathrm{~mm}$ ) in the axial plane parallel to the anterior-posterior commissure. During each functional run, about 310 contiguous axial images were acquired and the first two images were discarded because of saturation effects. Additionally, in order to improve the preprocessing and enhance the quality of the BOLD images ${ }^{58}$, T2 and T2* structural volumes were collected. Each functional and anatomical image was visually inspected to discard susceptibility artifacts and anatomical abnormalities.

Quality control and preprocessing were conducted using Statistical Parametric Mapping software (SPM12; Wellcome Trust Centre for Neuroimaging, London, United Kingdom) on Matlab 2017 (Mathworks Inc., Natick, MA, USA). Prior to the preprocessing, we manually centered the images to the AC-PC axis, realigned the functional and anatomical MRI images and then realigned all images to the last version of the SPM anatomical template "ch2". The preprocessing procedure was done with SPM12 and followed a methodology designed to reduce geometric distortion effects induced by the magnetic field, described by Villain and colleagues ${ }^{58}$. This procedure included the following steps: 1) realignment of the EPI volumes to the first volume and creation of the mean EPI volume, 2) coregistration of the mean EPI volume and anatomical T1, T2, and T2* volumes, 3) warping of the mean EPI volume to match the anatomical T2* volume, and application of the deformation parameters to all the EPI volumes, 4) segmentation of the anatomical T1 volume, 5) 
normalization of all the EPIs, T1 and T2* volumes into the Montreal Neurological Institute (MNI)

242 space using the parameters obtained during the T1 segmentation, 6) $8 \mathrm{~mm}$ FWHM smoothing of 243 the EPI volumes.

244 For each individual, frame-wise displacement (FD) ${ }^{59}$ was calculated. FD values greater than $2450.5 \mathrm{~mm}$ were flagged to be temporally censored or "scrubbed" during the first-level analysis (see 246 description below). The average of FD volumes censored was $M=6.8(S D=8.3$, Min $=1$, $\operatorname{Max}=$ 24738 ) for both runs for a total of $n=65$ participants. In addition, other $n=3$ participants were excluded 248 from further analysis because the number of volumes with FD $>0.5 \mathrm{~mm}$ exceeded $10 \%$ of the total 249 volumes acquired in one run.

\section{General linear model analysis with SPM}

The MRI SoVT-Rest data was analyzed using General Linear Models in SPM12 (implemented in Matlab 2017). This comprised a standard first-level analysis at the subject level, followed by a random effect (2nd-level) analysis to assess the effects of interest at the group level. For the 1stlevel analysis, a design matrix consisting of two separate sessions was constructed for each participant. Experimental event regressors in each session included the fixation cross (10 sec), instructions (4 sec), three videos ( $\sim 15 \mathrm{sec}$ each) modeled separately, and rest periods following each block $(90 \mathrm{sec})$. Each rest period was divided into three equal parts (30 sec time bins), in order to model different time intervals during which brain activity may gradually change after the end of the $\mathrm{HE}$ and LE video blocks (similar to Eryilmaz and colleagues ${ }^{24}$ ).

The different regressors were then convolved with a hemodynamic response function (HRF) according to a block design for univariate regression analysis. The six realignment parameters were added to the matrices in order to account for motion confounds, and low-frequency drifts were removed via a high-pass filter (cutoff frequency at $1 / 256 \mathrm{~Hz}$ ). The final 1st-level matrix consisted of 2 sessions of 21 regressors each (1 fixation cross +1 instruction for videos +1 instructions for rest +3 HE videos +3 post HE rest +3 LE videos +3 post LE rest +6 motion parameters) Additionally, to address the influence of remaining motion on BOLD data, we performed data censoring as described by Power and colleagues ${ }^{59}$. Specifically, during the estimation of beta coefficients for each regressor of interest, volumes with FD $>0.5 \mathrm{~mm}$ were flagged in the design matrices and ignored during the estimation of the $1^{\text {st }}$-levels.

For the 2nd-level analysis, we used a flexible factorial design where the estimated 272 parameters from 1st-level contrasts of interest were entered separately for each subject. The second273 level design matrix was generated with SPM12 and included 12 regressors of interest (3 HE videos 
+ 3 Post HE rest +3 LE videos +3 Post LE rest). This step allowed us to investigate the effect of each experimental condition on brain activity, including the main condition effects (video and rest) as well as the specific emotional effects (HE and LE) during either the video or the subsequent rest periods.

We then conducted T-tests contrasts to compare the conditions of interest (videos vs. rest periods and vice versa) as well as the specific emotional effects (videos: HE vs. LE; rest: HE vs. LE). We also identified voxels that were most reliably activated for a specific contrast (HE $>$ LE) across the two periods (videos and rest) by applying an inclusive masking from one contrast (e.g., videos: $\mathrm{HE}>\mathrm{LE}$ ) to the other contrast (rest: $\mathrm{HE}>\mathrm{LE})$ with a strict threshold used for both $(p<$ 0.00001). All comparisons are reported with a whole-brain family-wise error (FWE) correction at $p<0.05$, at the voxel level.

\section{Functional connectivity analysis during rest periods, definition of Regions of Interest} (ROI), and the data analysis pipeline

We conducted functional connectivity analyses between the most important brain regions of interest (ROIs) associated with the empathy network and with the default mode network (DMN). In addition, we also included the bilateral amygdalae among regions used for this analysis, because previous studies assessing carryover effects in the brain have related sustained amygdala activity to anxiety traits ${ }^{19}$ and emotional reactivity ${ }^{37}$. For nodes of the DMN, we choose the posterior cingulate cortex (PCC) and the anterior medial prefrontal cortex (aMPFC), following AndrewsHanna and colleagues ${ }^{60}$. Based on the results of a meta-analysis by Fan and colleagues ${ }^{44}$, the bilateral anterior insula (AI) and anterior medial cingulate cortex (aMCC) were used as ROIs in the empathy network. Time-series were extracted from $6 \mathrm{~mm}$-radius spheres around the peak of each of these ROIs. The amygdala was defined anatomically using the current SPM anatomical template provided by Neuromorphometrics, Inc (http://Neuromorphometrics.com/).

Functional connectivity analyses were performed using Matlab 2017 and R studio (version 3.6.1). For each participant, time courses of activity (from each voxel of the brain) were high-pass filtered at $256 \mathrm{~Hz}$, detrended and standardized (Z-score), before extracting specific time courses from the defined ROIs. Nuisance regressors included white matter (WM) and cerebrospinal fluid (CSF) signals, and the realignment parameters. For each participant, time-series from the instructions and videos periods were removed, and the remaining time series corresponding to the rest periods were concatenated. This procedure was previously proposed by Fair and colleagues ${ }^{61}$ and proved to be qualitatively and quantitatively very similar to continuous resting-state data. 
307 Additionally, in order to correct extreme head motion without affecting the autocorrelation of the 308 time series, image volumes flagged with FD $>0.5 \mathrm{~mm}$ were removed and replaced by interpolation 309 (every flagged volume $\mathrm{X}$ was replaced by the estimated mean of the $\mathrm{X}-1$ and $\mathrm{X}+1$ volumes). The 310 final concatenated time series resulted in 184 frames $(\sim 386 \mathrm{~s})$ of resting-state data for each subject.

311 We then correlated the time-courses between the different ROIs using Pearson correlations

31262 and the resulting coefficients were Fisher's $r$ to $z$ transformed in order to improve normality in 313 the data. Individual Z-score maps (correlation matrices) were created for each participant (see Fig. $3142 \mathrm{a}, \mathrm{b}, \mathrm{c}$ ). To test for significant differences between the two correlation matrices (post HE rest and 315 post LE rest), we used a non-parametric permutation test ${ }^{63}$. For each pair of nodes, the permutation 316 test compared the true correlation difference (e.g., HE - LE) to a null distribution built by randomly 317 flipping the sign of the correlation coefficients and computing the difference many times $(n=5000)$ 318 (see Fig. 2d). More precisely, for each pair of nodes (e.g., HE - LE for ROI 1 and ROI 3), a vector 319 of $n=127$ values was obtained and a one-sample $t$-test was computed to obtain the real $t$ value $(t$ 320 real). Then, the signs of the elements in the vector were randomly flipped $(n=5000)$ and the model 321 was fitted repeatedly once for every flipping. For each fit, a new realization of the $t$ statistic was 322 computed so that an empirical distribution of $t$ under the null hypothesis was constructed ( $\left.t_{\text {permuted }}\right)$. 323 From this null distribution, a $p$-value was computed by assessing the probability of the $t$ real to be 324 higher than $95 \%$ of the values on the empirical $t_{\text {permuted }}$ distribution ${ }^{63}$. Finally, the obtained $p$-values 325 were converted into an equivalent $Z$-score and significant changes were retained for $Z>1.64$ 326 (equivalent to $p<0.05$, one-tailed, uncorrected, marked by an asterisk in matrices). 


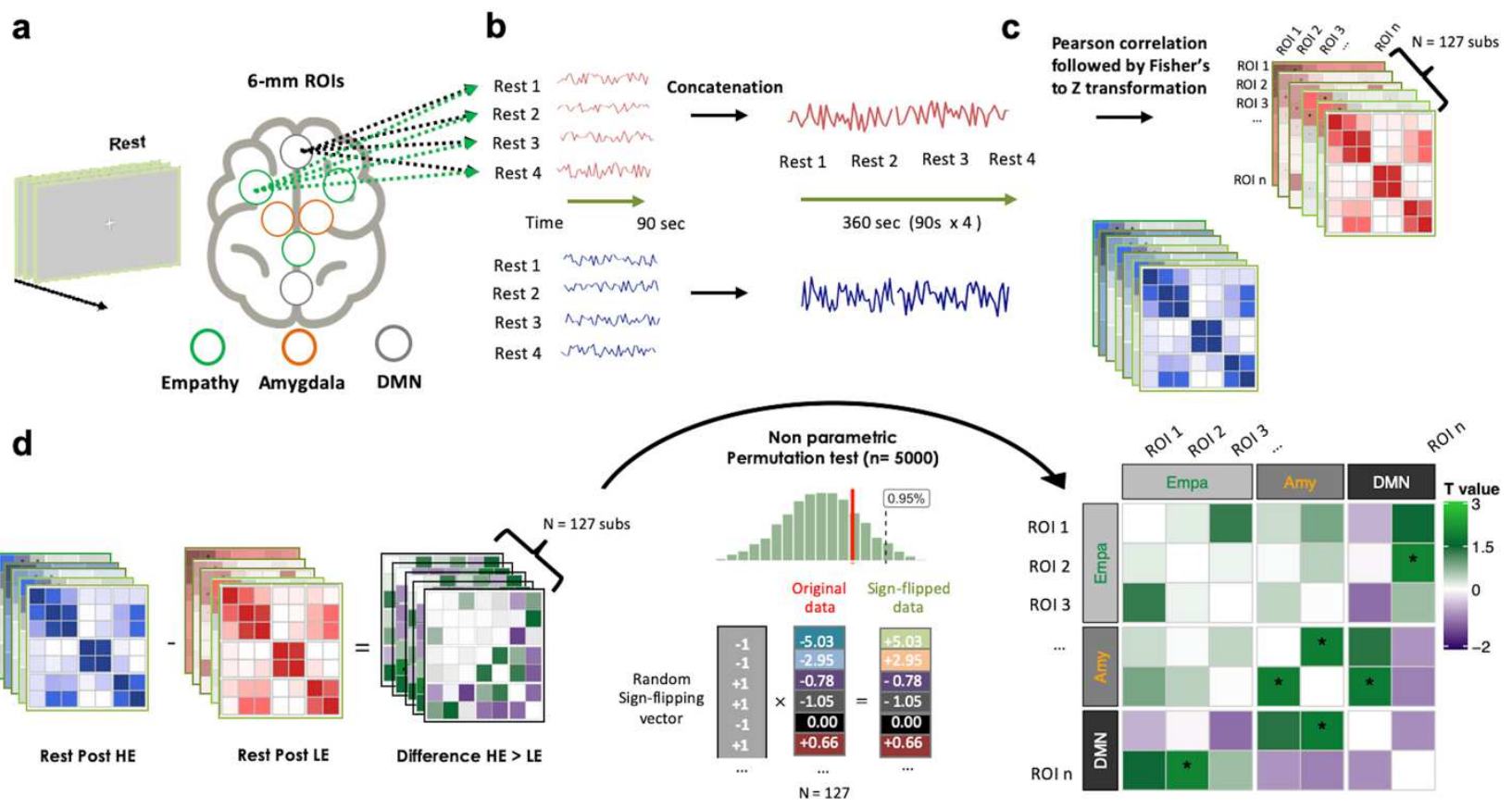

329 Figure 2. Functional connectivity pipeline: (a) Regions of interest (ROls) from the default mode network 330 (DMN) were chosen based on Andrews-Hanna et al. (2010), including the posterior cingulate cortex (PCC, $3318-5626$ ) and anterior medial prefrontal cortex (aMPFC, -6 $52-2$ ). ROls from the empathy network were 332 based on the meta-analysis by Fan et al. (2011), including the bilateral anterior insula (Al, -36 162 and 38 33324 -2) and anterior mid cingulate cortex (aMCC, -2 24 38). A 6 mm-radius sphere was created for each ROI. 334 The amygdala was defined anatomically using the SPM anatomical template. (b) For every participant, time335 series from the video and instruction periods were removed, and the remaining time series corresponding to 336 the rest periods were concatenated ${ }^{61}$. The final concatenated time series of the four rest blocks for each 337 type of video (high emotion, HE or low emotion, LE) resulted in 184 frames ( $360 \mathrm{~s}$ ) of resting-state data for 338 each subject. (c) We then correlated the time-courses between the different ROls using Pearson's $r$ 339 correlation, and the resulting coefficients were Fisher's $r$ to $z$ transformed to improve normality in the data. 340 Individual Z-score maps (correlation matrices) were created for each participant. (d) Finally, significant 341 differences between the two correlation matrices (post HE rest vs. post LE rest) were tested using a non342 parametric permutation test ${ }^{63}$. For each pair of nodes, the permutation test compared the true correlation 343 difference $t_{\text {real }}\left(\mathrm{HE}\right.$ vs. LE) to a null distribution $t_{\text {permuted }}$ constructed by randomly flipping the sign of the 344 correlation coefficients and repeating the $t$ statistic $(n=5000)$.

\section{Thought probes}

347 For each participant, two thought probes were recorded after the last rest period of each run (see 348 Fig. 1b), allowing us to test for differences in spontaneous mind wandering after emotional videos.

349 Following a transcription of the corresponding verbal recordings, the narratives of the participant's 350 responses were analyzed by two independent raters. For each probe (post HE rest and post LE rest), 
the raters attributed the presence (Present) or the absence (Absent) of specific thought contents according to a diverse set of pre-defined categories (Supplementary Table 2). These categories were selected according to a priori relevant affective or cognitive dimensions, and included the following: negative and positive emotions, directed attention to oneself and to others, emotion regulation (voluntary control of emotions), negative and positive social emotions, rumination, and temporality (present or past/future). Categories with low intra-group variability (i.e., less than $15 \%$ in one category) were not included in further analyses (for details, see Supplementary Table 2). The final dimensions included negative and positive emotions, directed attention to oneself and to others, and positive social emotions. This final analysis of thought probes comprised data from 109 participants for rest periods after HE videos and 110 participants for the rest periods after LE videos. This was due to i) missing thought probes for 9 participants and ii) exclusion of thought content that did not refer directly to the rest period, but rather to the video for both runs $(n=5)$, following LE rest ( $n=$ 3 ) or following HE rest $(n=4)$. Interrater agreement on the final dimensions ranged from 0.28 to 0.66 (Cohen's kappa index; see Supplementary Table 2 for details). The statistical analyses were performed with R studio (version 3.6.1) and the corresponding graphs were created with ggplot2 (version 3.2.1).

\section{Results}

\section{Independence of the three parallel video sets of the SoVT-Rest task}

To check whether the three video sets elicited similar emotions in this elderly sample, we performed a repeated measures multivariate analysis of variance (MANOVA, with Pillai's trace statistics) with the within-subject factor video type (HE vs LE), the between-subject factor video set (V1, V2, V3), and three dependent variables: empathy, positive affect, and negative affect ratings. As expected, and replicating results from Klimecki and colleagues ${ }^{48}$, this analysis revealed no significant differences between the three video sets for any of the self-reported ratings (Pillai's trace $=0.01$, $F(1,125)=0.55, p=0.6)($ see Fig. 3a).

\section{Reliable impact of high compared to low emotion on affective and empathy ratings}

We compared the effects of $\mathrm{HE}$ and LE videos using pairwise $t$-tests for each of the three affective ratings (empathy, positive, and negative affect). As predicted, participants reported higher levels of empathy $\left(t_{126}=14.5, p<0.001, d=1.31\right.$, two-tailed $)$, higher negative affect $\left(t_{126}=26.9, p<0.001\right.$, 
$382 d=2.89$, two-tailed $)$, and lower positive affect $\left(t_{126}=-18.9, p<0.001, d=-2.31\right.$, two-tailed $)$, when 383 presented with $\mathrm{HE}$ as compared to LE videos (see Fig. 3a). These data validate a successful 384 elicitation of socio-emotional responses with the SoVT-Rest.

\section{Correlation between empathy and affective valence during the SoVT-Rest}

387 To test how empathy was associated with positive and negative affect during HE and LE videos, 388 we computed Spearman correlations between these rating scales. These analyses revealed that 389 during HE videos, empathy correlated positively with negative affect ( $r h o=0.61, p<0.001)$ and 390 negatively with positive affect ( $r h o=-0.36, p<0.001$ ). Interestingly, during LE videos, empathy 391 also correlated positively with positive affect $(r h o=0.62, p<0.001)$ but not with negative affect $392 \quad($ rho $=0.08, p<0.35)$ (see Fig. 3b).

\section{Correlation between emotional responses to the SoVT, age, and personality traits}

395 To assess whether levels of empathy, positive affect, and negative affect elicited by the SoVT varied 396 with age and questionnaire measures of empathy, depression, anxiety, rumination and emotion 397 regulation, we conducted additional Spearman rank correlations followed by multiple comparison 398 corrections (using False Discovery Rate). As shown in Figure 3c, this revealed that participants who 399 experienced higher levels of negative emotions during HE videos also reported higher levels of trait 400 anxiety $(r h o=0.24, p=0.006)$ and empathic concern (subscale of the Interpersonal reactivity index; 401 IRI) (rho $=0.28, p=0.001$ ). Empathy during HE videos was also positively correlated with 402 empathic concern on the IRI ( $r h o=0.26, p=0.002)$. Interestingly, age was correlated with positive 403 emotions $(r h o=0.26, p=0.002)$ and negatively correlated with negative emotions $(r h o=-0.21, p$ $404=0.01$ uncorrected) (see Fig. 3c). For completeness, correlations that did not survive correction for 405 multiple comparisons are also displayed in Fig. 3c. 
a
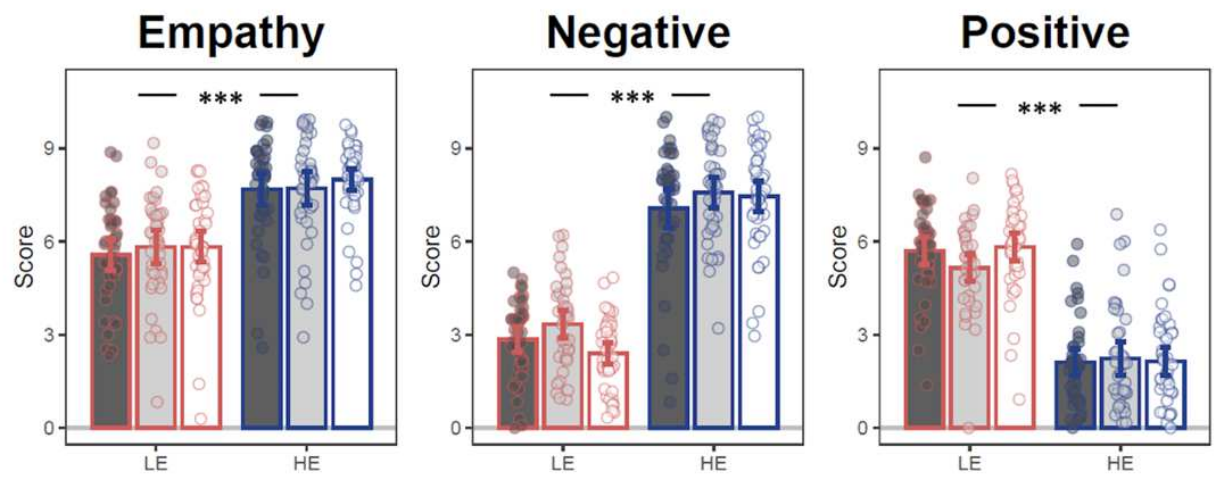

$* * *=p<0.001$

Videoset

$\begin{array}{ll}\begin{array}{ll}\text { aㅣ V1 } \\ \text { O V }\end{array} & \text { V2 } \\ 0 & \text { V3 }\end{array}$

Video Type

Low Emotion

High Emotion

b
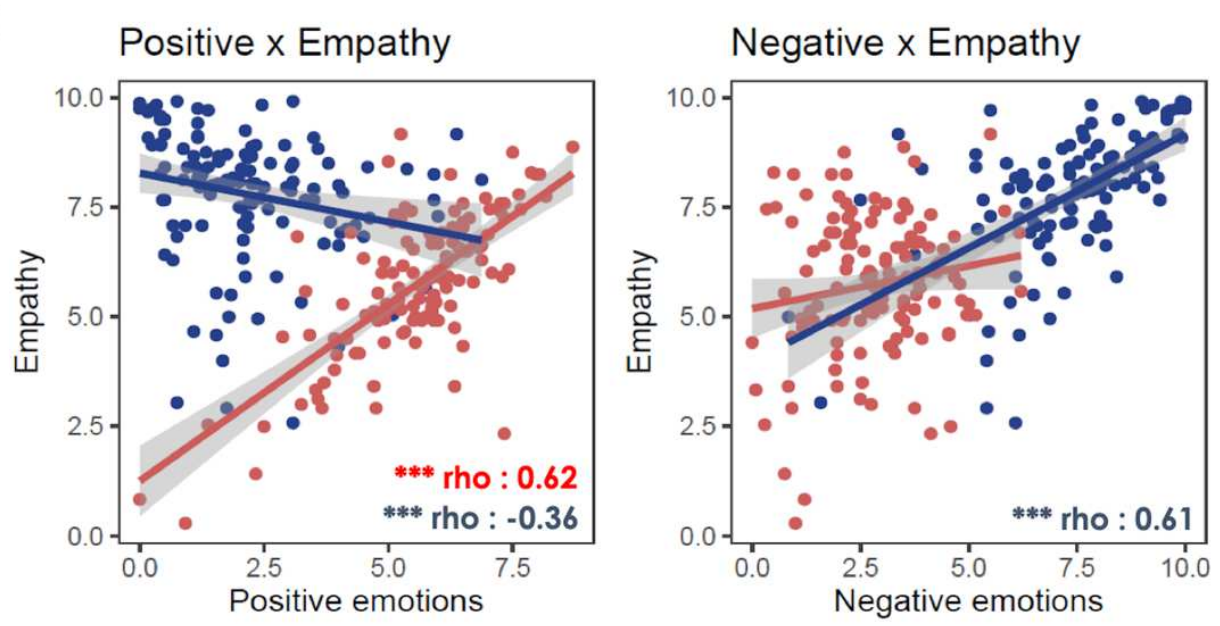

$* * *=p<0.001$

Video type

Low Emotion

High Emotion

C

Personality traits

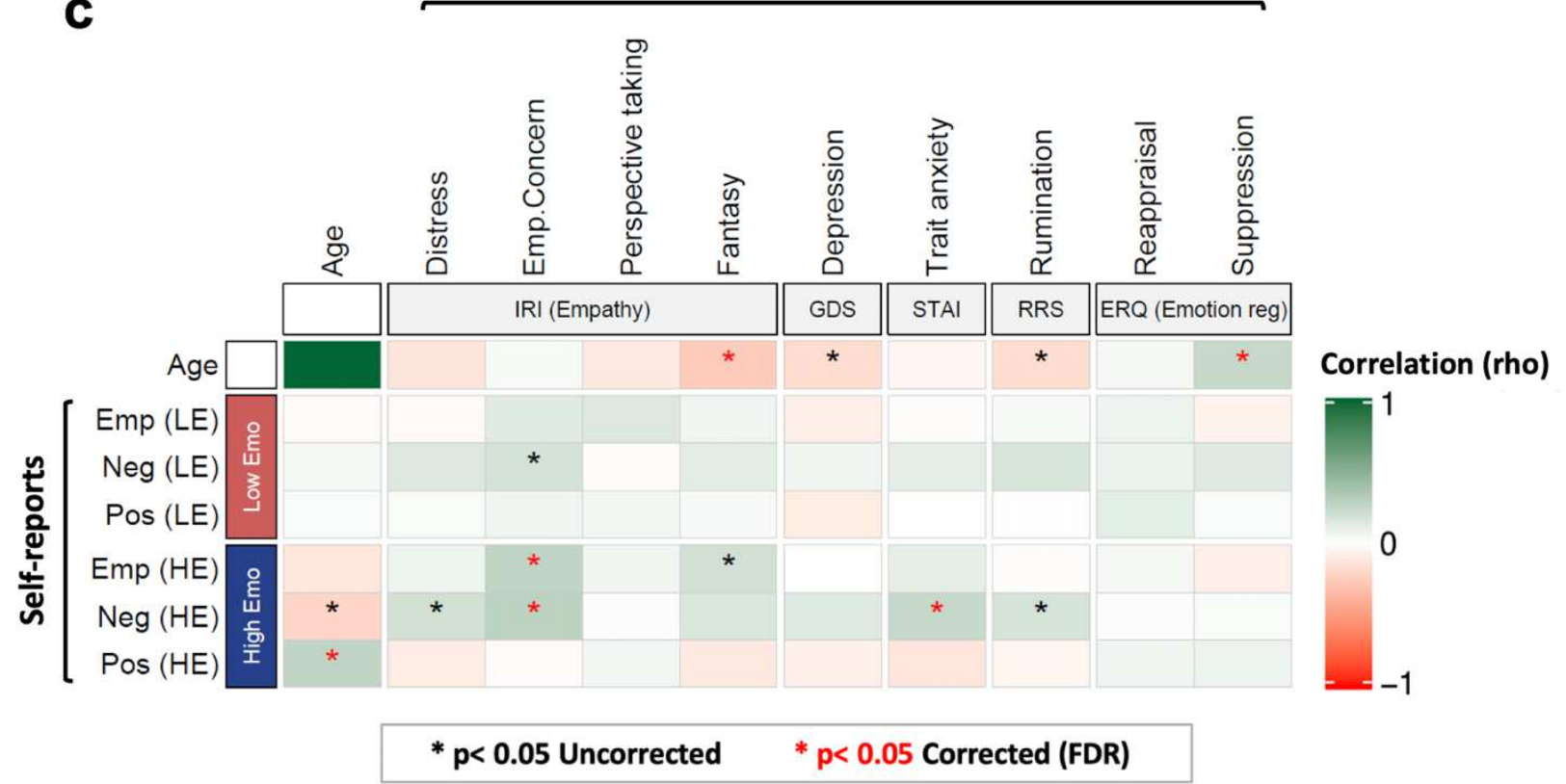

Figure 3. (a) Self-reported scores of empathy, positive affect, and negative affect for the HE and LE videos.

408 (b) Spearman correlations between self-reported scores of empathy and affective ratings. Error bars 409 represent 95\% confidence intervals; dots represent averaged values for each participant per condition, $n=$ 
410 127. (c) Spearman correlations between age, personality traits, and self-reported scores of empathy, positive 411 affect, and negative affect. Blue: HE videos, Red: LE videos, $n=126$ (1 missing data point). IRI: Interpersonal 412 Reactivity Index, GDS: Geriatric Depression Score, STAl: STAl-trait Anxiety Index, RRS: Rumination

413 Response Scale, ERQ: Emotion Regulation Questionnaire.

414

\section{Main effects of videos and rest periods (manipulation check)}

416 Regarding the neuroimaging results, we first verified that the video and rest periods induced 417 differential brain activity by testing for the main effects of task condition. As expected, comparing 418 videos versus rest periods (Videos $>$ Rest, voxel-wise $p<0.05$ FWE-corrected) revealed greater 419 activity in widespread networks, including strong increases in visual cortices. On the opposite, 420 comparing rest versus video watching periods (Rest $>$ Videos, voxel-wise $p<0.05$ FWE-corrected) 421 revealed greater activity in several regions typically associated with the default mode network, such 422 as the PCC/Precuneus, ACC/MPFC, and bilateral IPL (see Supplementary Fig. 1 and 423 Supplementary Table 3).

424

425 Main brain regions activated when faced with others' suffering during HE videos

426 We then determined the effect of the emotional content of videos (high vs low). The contrast of HE $427>$ LE conditions (voxel-wise $p<0.05$ FWE-corrected) showed that HE videos (people who are 428 suffering) induced greater activity in several brain areas including bilateral anterior insula (AI), left 429 temporo-parietal junction (1. TPJ), posterior and anterior mid-cingulate cortex (PCC, aMCC), dorsal 430 medial prefrontal cortex (dMPFC), and ventral striatum (VS) (see Fig. 4). These areas overlap with 431 brain networks classically associated with empathy ${ }^{35,44}$, compassion ${ }^{48,56,64}$, as well as cognitive 432 and affective theory of mind ${ }^{65,66}$. The opposite contrast (LE $>$ HE) showed no significant activation. 433 


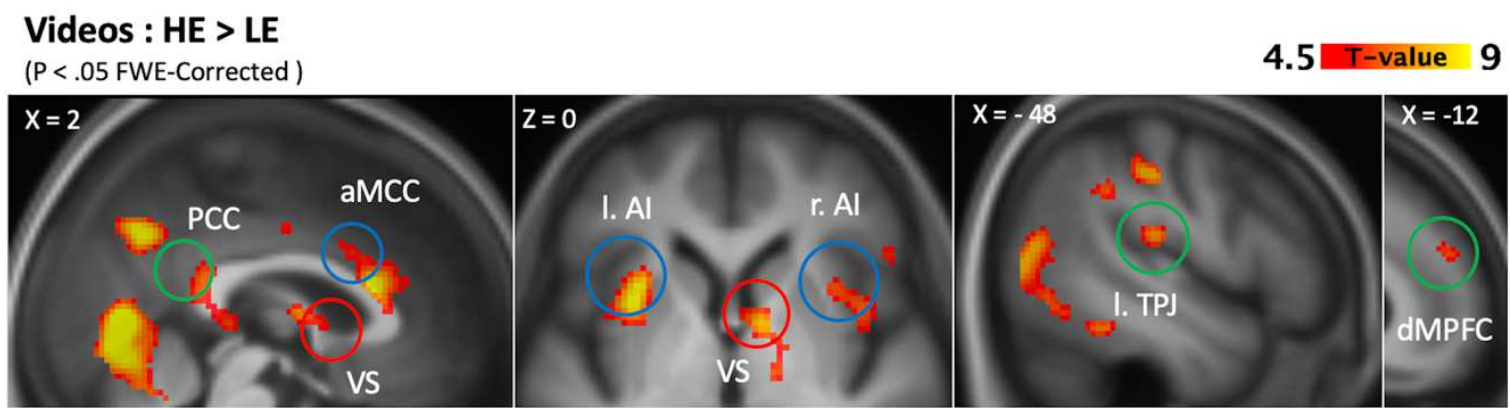

435 Figure 4. Brain regions with greater activation during high emotion (HE) videos in contrast to low emotion 436 (LE) videos. Reported results are corrected for multiple comparisons using familywise error (FWE) correction 437 at the voxel level $(p<0.05 \mathrm{FWE}$-corrected). Blue circles show regions previously reported as part of the 438 Empathy network (bilateral anterior insula, Al; anterior middle cingulate cortex, aMCC), green circles show 439 regions previously reported as part of the Theory of Mind network (PCC: posterior cingulate cortex, I. TPJ: 440 left temporo-parietal junction, dMPFC: dorsal medial prefrontal cortex), red circles show regions associated 441 with the Compassion network (VS: ventral striatum) ${ }^{64,67}$. Activations are displayed on the average T1 image 442 of our 127 participants.

\section{Carryover effects of emotional videos observed in the subsequent rest periods:}

445 To test for carryover effects of emotional videos on subsequent resting states ${ }^{24}$ and thus assess 446 homeostatic emotion regulation abilities ${ }^{16}$, we compared rest periods after HE videos to rest periods 447 after LE videos (post HE $>$ post LE; voxel-wise $p<0.05$ FWE-corrected). This contrast revealed 448 greater brain activations in two main areas among the midline nodes of the DMN (ACC/MPFC, and 449 Precuneus/PCC), as well as greater activations of the right amygdala (r.AMY) and the ventral part 450 of the right anterior insula (r.AI) (see Fig. 5a). This accords with previous evidence of emotional 451 inertia effects on brain activity and DMN in younger adults ${ }^{24}$.

452 We then asked whether these carryover effects directly resulted from higher activity in these 453 regions during HE videos. To this aim, we use a masking procedure (see Methods) to overlap the 454 emotional increases (contrasts $\mathrm{HE}>\mathrm{LE}$ ) from both the videos and the rest periods and determine 455 common areas of activity, shared across the task conditions (Fig. 5a). This comparison revealed a 456 restricted overlap in a few selective regions, mainly dMPFC and PCC, where voxels with emotional 457 activation during videos also exhibited emotional carryover effects at rest after videos, suggesting 458 sustained increases persisting over time (Fig. 5c,d). In contrast, other regions differentially activated 459 during emotional videos did not display any carryover effects during the subsequent rest periods 460 (i.e., exclusively responding to $\mathrm{HE}>\mathrm{LE}$ conditions during the videos periods), including not only 
461 visual cortical areas but also mid cingulate areas (MCC; Fig. 5b). Interestingly the right amygdala 462 as well as a segment of the right anterior insula (ventral part) did not show significant differences 463 for the $\mathrm{HE}>\mathrm{LE}$ contrast during videos but were robustly activated in the post $\mathrm{HE}>$ post LE rest 464 periods (Fig. 5e,f). These dissociations between rest and video-related activity are further illustrated 465 by plots of brain activity (contrasts estimates) over time across the different task periods (using a 466 single time bin of $\sim 45 \mathrm{sec}$ during videos and three successive time bins of 30 seconds during rest to 467 depict the time course of the activation) and the different conditions (HE and LE videos) (Fig. 5).

468 The opposite contrast (post LE $>$ post HE; voxel-wise $p<0.05$ FWE-corrected) revealed 469 increased activations in regions including the left parahippocampal gyrus (1.PHG) and the right 470 superior/middle frontal gyrus (r.SFG) (see Supplementary Fig. 2 and Supplementary Table 3). 471 These regions have been previously associated with environmental scenes processing ${ }^{68}$, spatial 472 navigation ${ }^{69}$, and working memory ${ }^{70}$. 

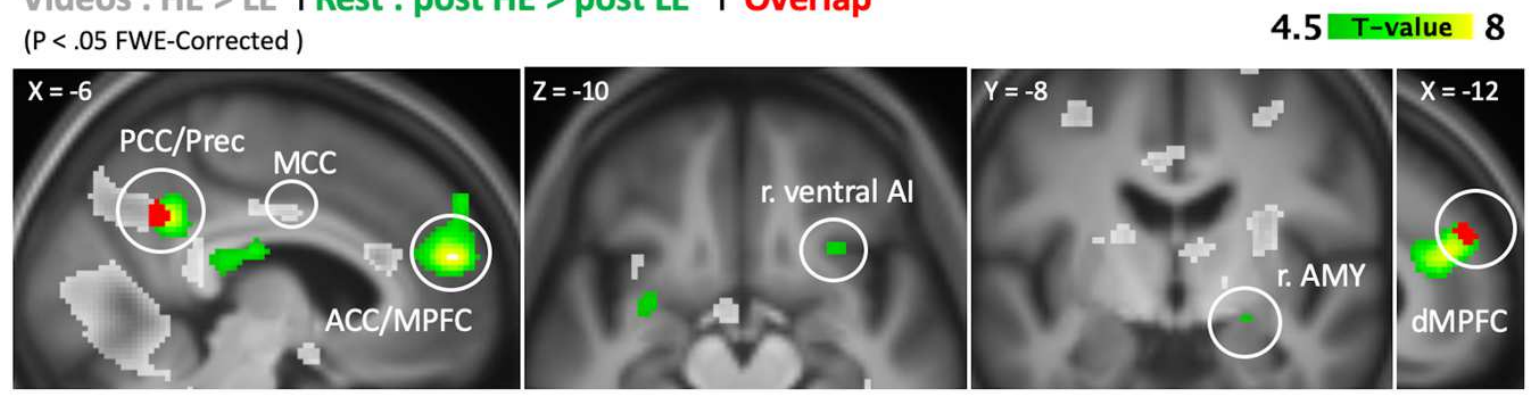

b

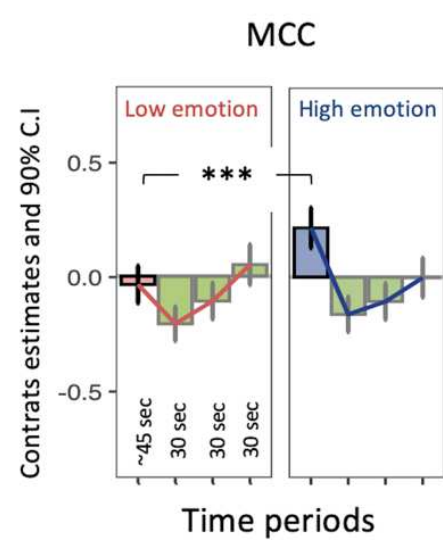

e

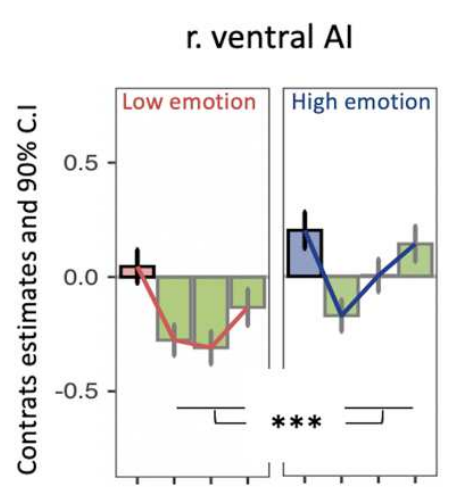

Time periods
C PCC/Prec

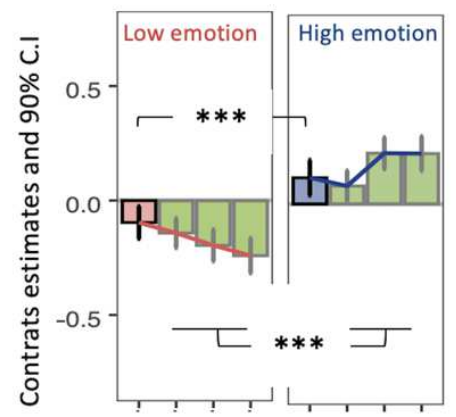

Time periods

f

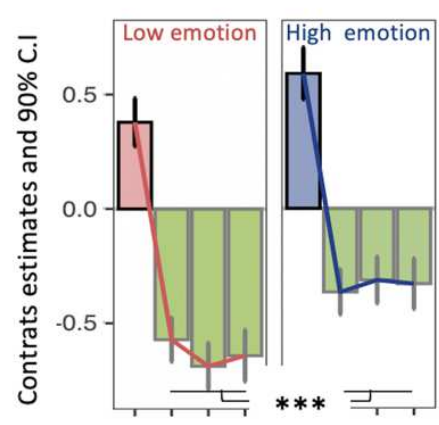

Time periods d $\quad$ dMPFC

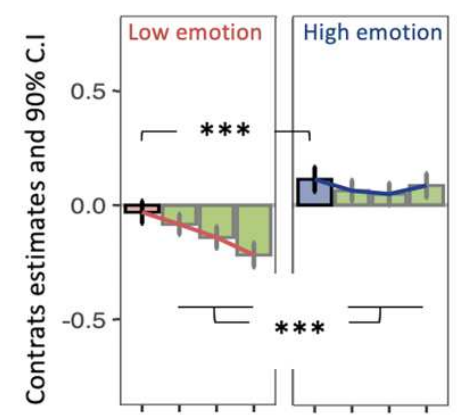

Time periods

Videos periods

Rest periods

478 Figure 5. Brain activations to high emotion (HE) versus low emotion (LE) videos and corresponding carryover effects during rest periods. (a) Clusters in grey show brain regions significantly activated in the contrast HE videos > LE videos. Clusters in green show brain regions significantly activated in the rest periods corresponding to the contrast post HE videos > post LE videos. Red clusters show the overlap.

482 Reported results are $p<0.05$ corrected for multiple comparisons using family-wise error (FWE) correction 483 at the voxel level. (b,c,d,e,f) Magnitude and time-course of brain activity (parameter estimates) for relevant regions during the different task periods. (b) Example of a region (in MCC) responding to HE vs LE videos, but showing no significant difference in activation during rest after HE vs LE videos. (c,d) Example of regions (PCC/Prec and dMPFC) responding to HE > LE videos and showing significant carryover with sustained activity during subsequent rest. (e,f) The right amygdala as well as the ventral part of the right anterior insula 
corresponding rest. Pink lines track activity time-courses during LE conditions, blue lines track activity timecourses during HE conditions. Pink and blue bars indicate activity (blocks of 3 videos $=\sim 45$ seconds) for LE and $\mathrm{HE}$ videos respectively, green bars indicate activity (over 3 bins of 30 seconds) during rest periods subsequent to corresponding videos periods. Activations are displayed on the average T1 image of our 127 participants. ${ }^{* * *} p<0.05$ FWE-corrected. PCC: posterior cingulate cortex, Prec: precuneus, MCC: midcingulate cortex, ACC: anterior cingulate cortex, MPFC: medial prefrontal cortex, dMPFC: dorsal medial prefrontal cortex, r. ventral Al: right anterior insula (ventral part), r. AMY: right amygdala.

\section{Exposure to others suffering impacts subsequent brain network connectivity}

To further assess the lingering impact of emotional videos on brain activity dynamics (emotional inertia), we then examined differences in functional connectivity between and within a priori defined networks. To do so, we first determined the functional connectivity patterns in regional time-series from the default mode network, the empathy network, and bilateral amygdala measured during the rest periods after HE videos, compared to the rest periods after LE videos (Fig. 2). We computed connectivity matrices using Pearson correlations between the time-series of every pair of nodes in the three networks of interest. The resulting connectivity matrices obtained for each participant were then group-averaged for illustration (see Fig. 6a).

These brain connectivity maps revealed a general intra-network connectivity (Emp-Emp, Amy-Amy, DMN-DMN) during rest periods subsequent to both the HE and LE videos (Fig. 6a), consistent with functionally coherent activity within each specific network. To specifically unravel the differential connectivity during rest periods due to emotional inertia (post HE vs post LE rest periods), we directly compared the two connectivity matrices using permutation tests (see methods). Significant differences were observed for highly selective functional connections of the DMN with limbic areas: In contrast to rest periods after LE videos, rest periods after HE videos induced stronger functional coupling between the PCC and the right amygdala $(t=1.82, p=0.03, Z=1.81$ one-tailed), as well as between the aMPFC and left insula $(t=1.98, p=0.02, Z=2.02$ one-tailed). In addition, there was also higher coupling of the bilateral amygdala during rest periods after $\mathrm{HE}$ vs LE videos (right with left, $t=1.88, p=0.02, Z=1.95$ one-tailed) (Fig. 6b, 6c).

\section{Relationship between functional connectivity patterns and personality measures}

519 Our fMRI analyses identified a selective impact of emotional videos on functional brain 520 connectivity of the posterior part of the DMN (PCC) with the right amygdala (r.AMY) as well as 521 of the anterior part of the DMN (aMPFC) with the left anterior Insula (1.AI) at rest, providing a 522 plausible neural marker of emotional inertia ${ }^{16,24}$. To test whether these effects are related to 
523 individual differences in socio-affective processing and personality traits, we computed a further 524 correlation analysis between the $Z$-values from significant edges in connectivity matrices (i.e., 525 connections between two ROIs showing a significant difference $Z>1.64$ between post HE vs post 526 LE rest) and specific scores on the trait anxiety (STAI-trait), rumination (RRS), and empathy (IRI). 527 The results showed a significant positive relationship between the strength of changes in the PCC528 r.AMY connectivity (rest HE - rest LE) and the individual scores of trait anxiety $(r=0.21, p<$ 5290.01 , two-tailed) and rumination ( $r h o=0.22, p<0.01$, two-tailed) (Fig. 7). 

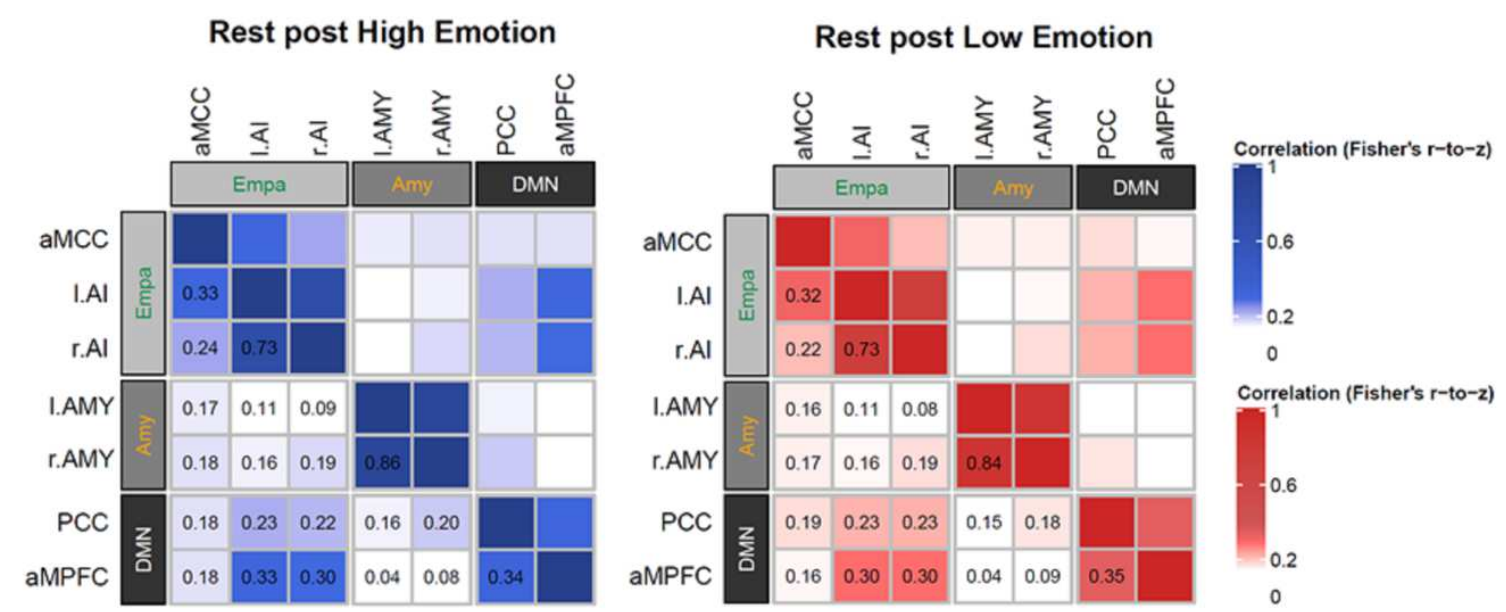

b

Rest: post HE - post LE

\section{Increased functional connectivity for \\ Rest: post HE - post LE}
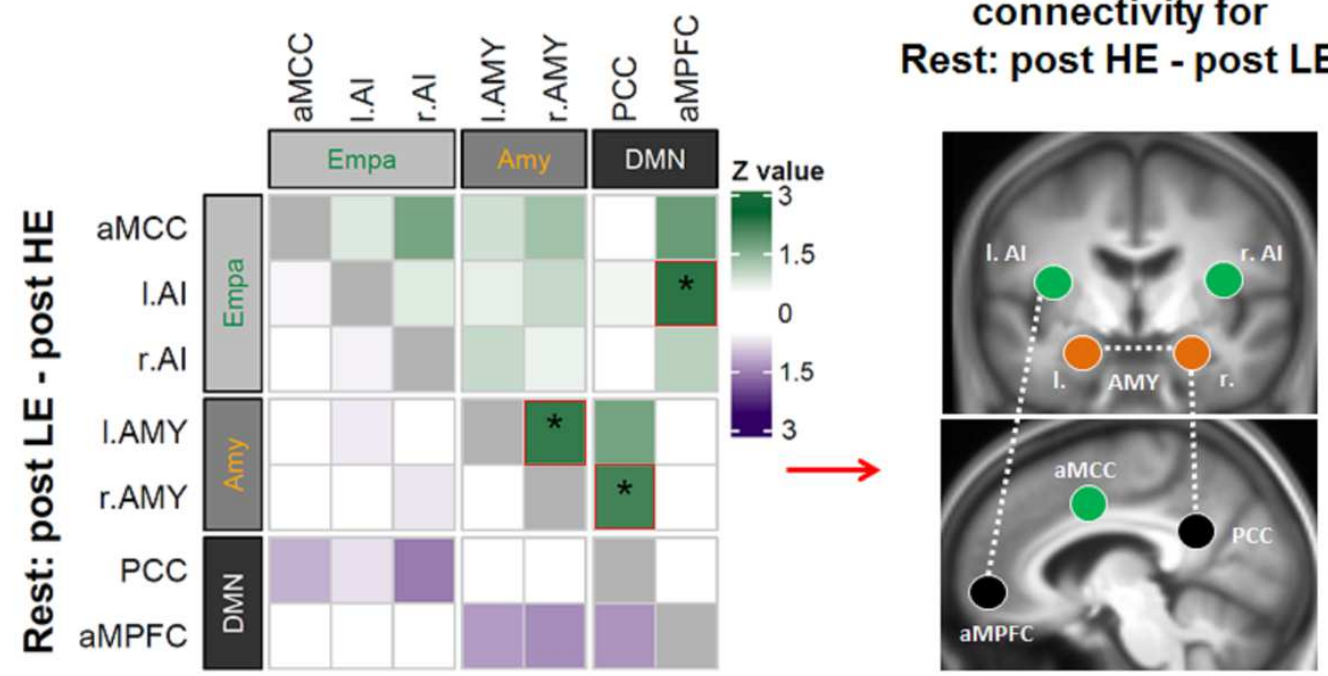

533 Figure 6. Functional connectivity results illustrated as (a) correlation matrices between pairs of ROls for

534 the different rest conditions. Blue matrix corresponds to post HE (high emotion) rest periods, red to post LE 535 (low emotion) rest periods. The plotted values (correlation coefficients following Fisher's $r$-to- $z$ 536 transformation) were obtained by averaging the $n=127$ correlation matrices for each condition.

537 (b) Correlation matrix corresponding to the difference between the two rest conditions, showing post emotion 538 increases (green) and post emotion decreases (violet). Left and right halves of the matrix with respect to the 539 diagonal depict the values for inverse contrasts (upper part of the matrix: post HE - post LE rest periods; 540 lower part of the matrix: post LE - post HE rest periods. Significant changes in correlations with $Z>1.64$ are 541 marked by an asterisk * corresponding to $p<0.05$, one-tailed uncorrected). (c) Visual representations of 542 significant changes in functional connections. Black ROIs= DMN regions, Orange ROIs = bilateral amygdala, 543 Green ROIs = empathy network regions. Al: anterior insula, aMCC: anterior mid-cingulate cortex, AMY: 544 amygdala, PCC: posterior cingulate cortex, aMPFC: anterior medial prefrontal cortex. The brain image 545 corresponds to the average of the 127 T1 images of our sample. 

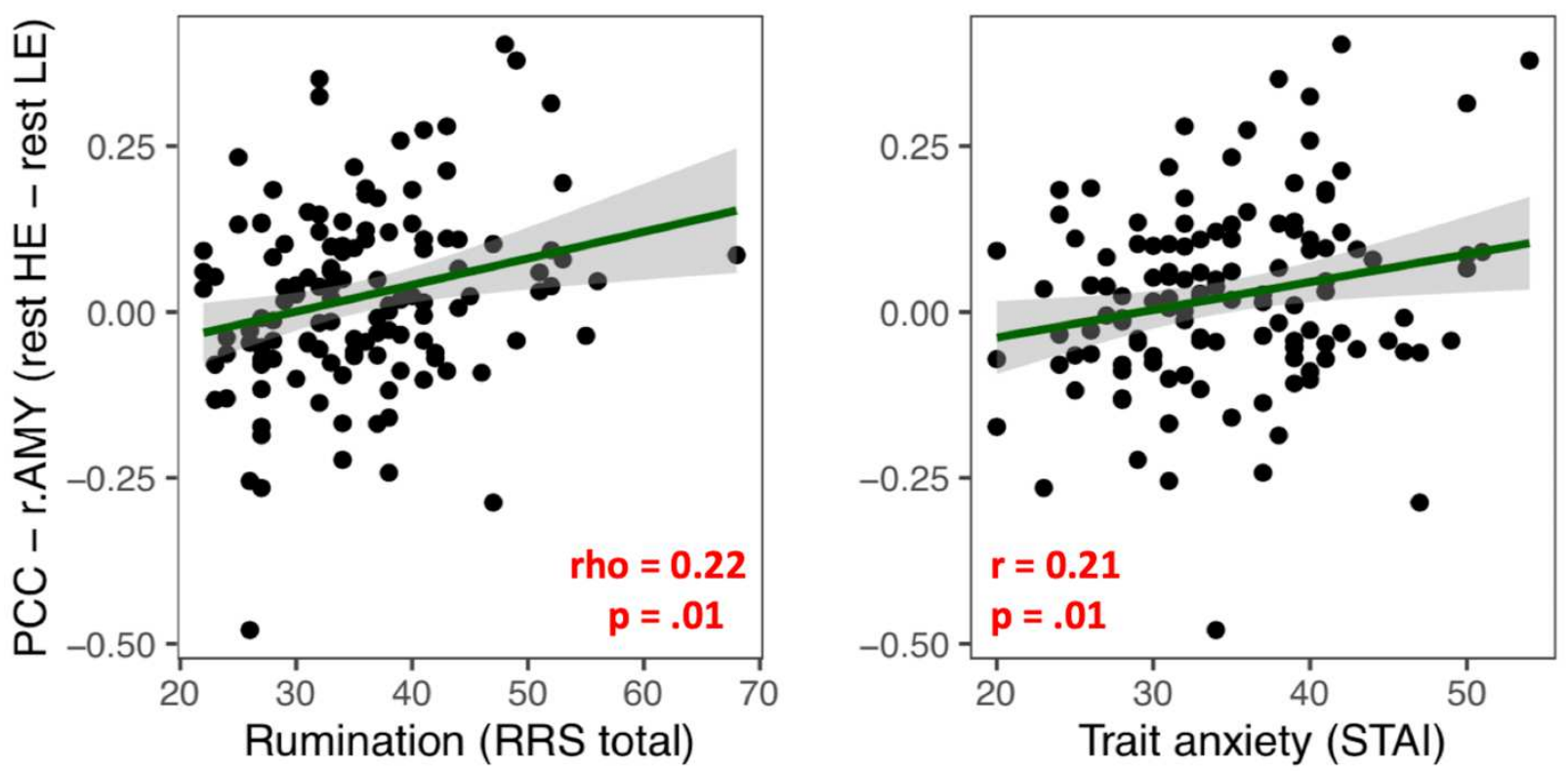

547 Figure 7. Pearson $(r)$ and Spearman $(r h o)$ correlations show that higher functional connectivity between 548 posterior cingulate cortex and amygdala during rest periods after HE > LE videos [PCC-r.AMY(rest HE-rest LE)] was positively related to trait anxiety (STAI.B) and rumination (RRS total).

\section{Relationship between functional connectivity patterns and thought probes}

Because we observed that rumination scores were positively associated with greater changes in

554 functional coupling between PCC-r.AMY for the contrast post HE > post LE at rest, we reasoned that some participants (i.e., with higher ruminative tendencies) may have kept more negative-related content in their thoughts during the rest periods after emotional videos. This was directly tested using the explicit thought probe given after different rest conditions (see Fig. 1b). To do so, we compared the PCC-r.AMY connectivity between a subgroup of participants who verbally reported negative content in their spontaneous thoughts in response to the probe question (Present) vs. those who did not (Absent), for both the HE and LE conditions.

Behaviorally, for rest periods after HE videos, 59(54\%) participants reported negative

562 thought content, while 30(28\%) reported no negative thought content and 20(18\%) were ambiguous

563 (judgments by our two raters did not match). Interrater reliability analyses revealed a good 564 agreement $(\mathrm{kappa}=0.61)$ between the two independent raters (see Supplementary Table 2 for 565 details). A Chi-square test revealed that these proportions (negative present $54 \%$ vs. negative absent $28 \%$ ) were statistically different; $X^{2}(1, N=109)=45.88, p<0.001$ (two-tailed), demonstrating that $\mathrm{HE}$ videos induced more frequent negative than non-negative thoughts in our participants. 
Conversely, for rest periods after LE videos, only 41(37\%) participants reported negative

569 thought contents, while 50(45\%) reported no negative thoughts, and $19(17 \%)$ were considered 570 ambiguous. The rater agreement was again good (kappa $=0.66)$ (see Supplementary Table 2 for 571 details). This proportion of negative thoughts (37\%) was significantly lower than the proportion of 572 non-negative thoughts $(45 \%)$; Chi-squared test, $X^{2}(1, N=110)=51.59, p<0.001$ (two-tailed), 573 indicating that the LE videos induced less frequent negative mental thought content (than non574 negative thoughts). An additional McNemar's test further determined that, as expected, participants 575 reported more negative thoughts for rest periods after $\mathrm{HE}$ videos than for rest periods after LE 576 videos, $X^{2}=10.28, p=0.02$ (two-tailed).

577 Finally, to relate these behavioral indices to brain effects, we used a non-parametric 578 permutation analysis in which the PCC-r.AMY connectivity difference (observed diff $=0.08$ ) 579 between these two subgroups (negative thoughts Present-Absent) was compared to a null580 distribution built by permuting the labels 5000 times. As hypothesized, we found that $54 \%$ of the 581 participants reporting negative content in their thoughts (vs. 28\% not reporting) showed increased 582 PCC-r.AMY connectivity for the rest periods following HE videos ( $p=0.02$, one-tailed). The same 583 difference between the two subgroups for rest periods following LE videos was only a trend 584 (observed diff $=0.06 ; p=0.07$, one-tailed) (Fig. 8a,b). Taken together, these findings further unveil 585 a direct relation between PCC-r.Amy connectivity changes after negative emotions and individual 586 reactivity to aversive or stressful socio-emotional stimuli. 
a

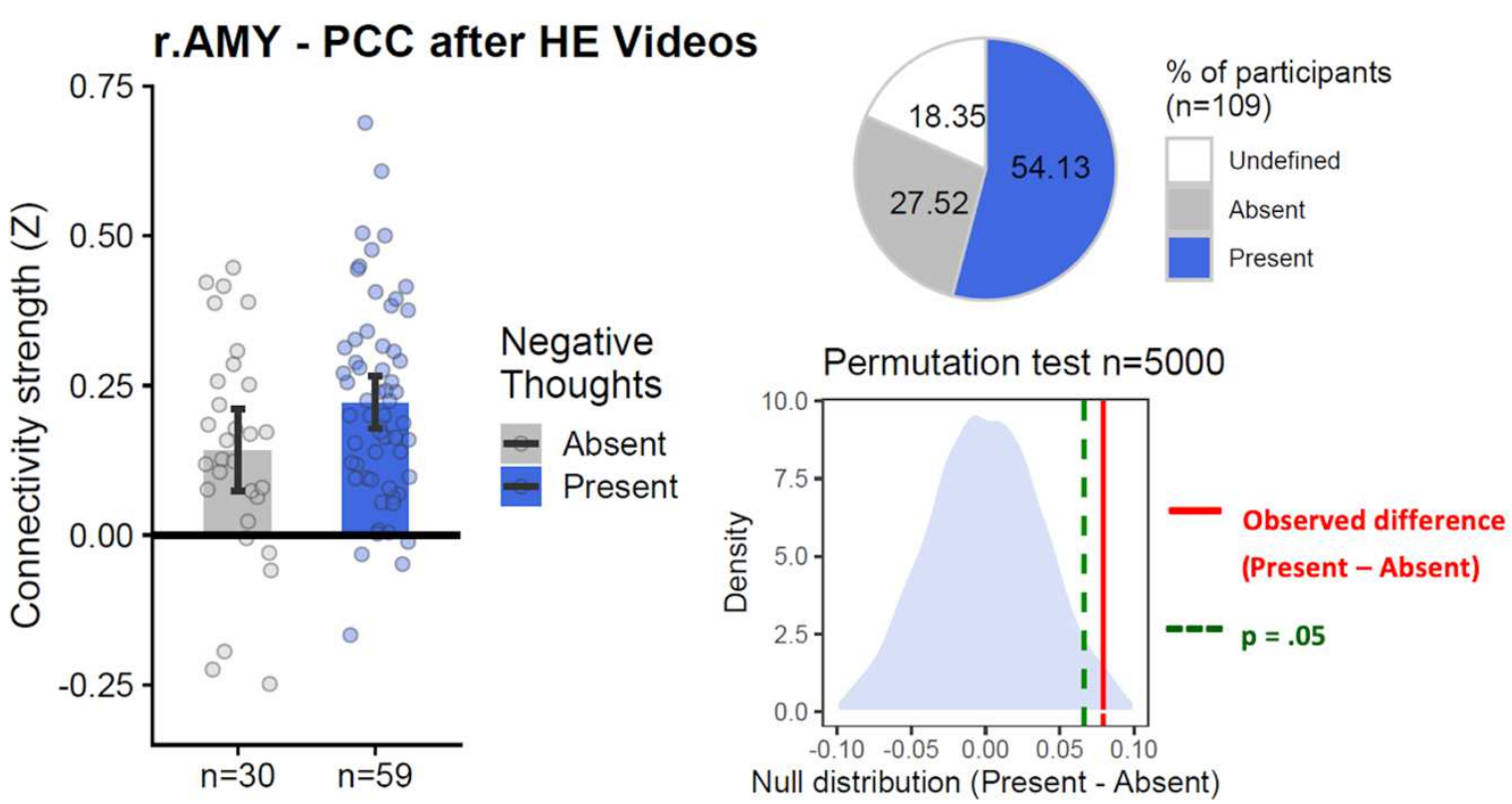

b
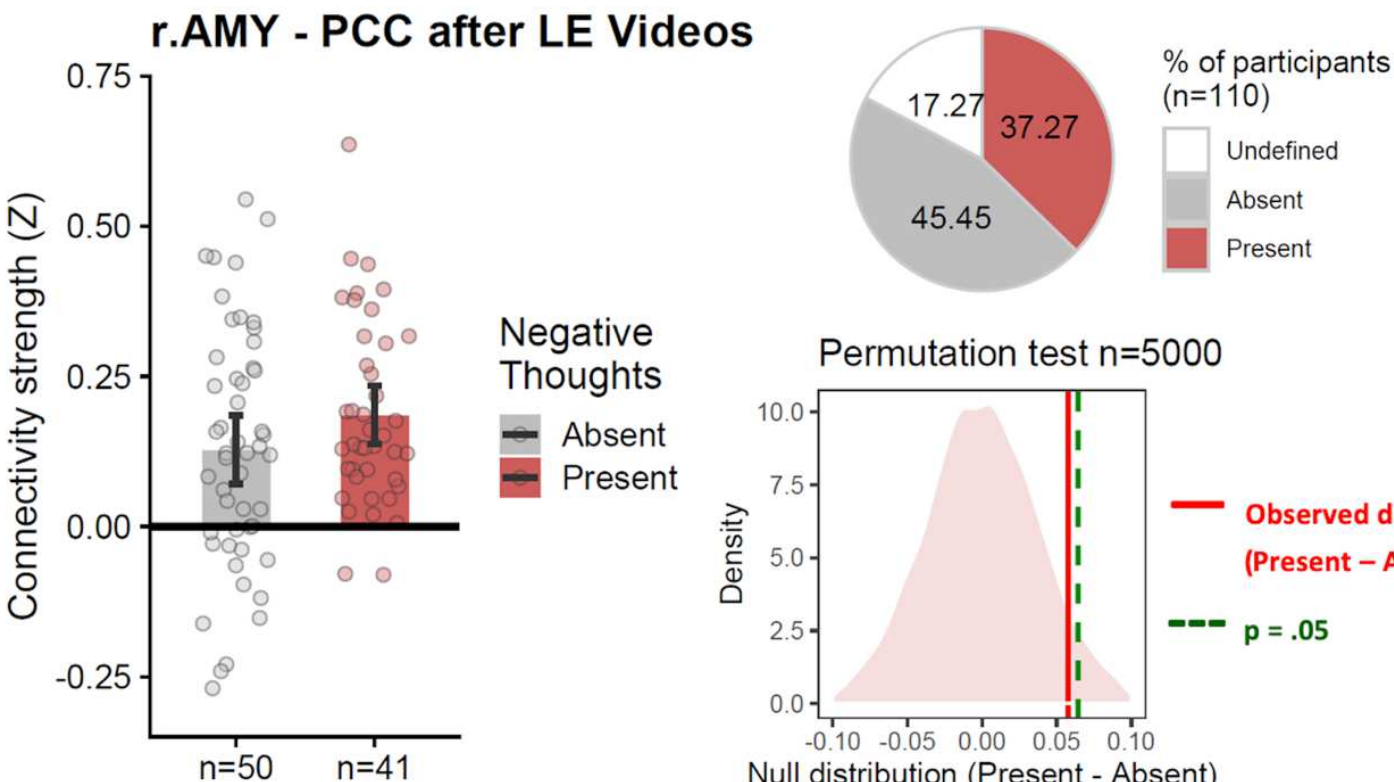

Figure 8. (a,b) r.AMY-PCC connectivity between the group of participants that verbally reported negative content during the thought probes (Present) vs. the group that did not (Absent), for both HE and LE conditions. After HE videos, 59(54\%) participants reported negative content in their thought probes, 30(28\%)

591 did not report negative content and 20(18\%) were ambiguous(undefined). After LE videos in turn, only $59241(37 \%)$ reported negative content vs. 50(45)\% not reporting negative thoughts and 19(17\%) were

593 ambiguous (undefined). At the brain level, the comparison was made with a non-parametric permutation

594 analysis in which the true mean PCC-AMY connectivity difference between the groups Present-Absent 595 (observed diff $=0.08$ ) was compared to a null-distribution built by permuting the labels 5000 times. As 596 hypothesized, we observed that $54 \%$ of the participants reporting negative content in their thoughts (vs. the $59728 \%$ not reporting) showed increased PCC- r.AMY connectivity in the HE conditions ( $p=0.02$, one-tailed). 
In the LE conditions, there was no significant difference in PCC- r.AMY connection between the two groups (observed diff $=0.06 ; p=0.07$, one-tailed). Blue: High Emotion (HE) condition, Red: Low emotion (LE) condition. r.AMY-PCC: connectivity between the right amygdala and the posterior cingulate cortex. The percentages in the text are rounded.

602

603

604

\section{Discussion}

The current study aimed at delineating neural markers of proficient emotional resilience and empathy in ageing, which are increasingly recognized as important protective factors against mental illness and cognitive decline in this population ${ }^{71}$. We assessed both reactivity and recovery of brain networks to negative socio-affective situations (i.e., during and after exposure) in a large cohort of healthy elderly, allowing us to probe for emotional carryover effects in resting state (emotional inertia) as an indicator of maladaptive regulation processes ${ }^{16}$, and to examine their relationship with measures of anxiety, rumination, and negative thoughts.

614 Our behavioral and imaging results extend previous findings in younger adults ${ }^{48}$. On the behavioral 615 level, the present data confirm that seeing others' suffering induced higher levels of negative affect, 616 lower positive affect, and higher empathy scores than mundane scenes of daily life in the elderly, 617 albeit at different levels (see Supplementary Fig. 3 for a comparison with young participants from 618 Klimecki and colleagues ${ }^{48}$. As expected, negative affect generally correlated with higher anxiety 619 and rumination levels in our participants (see Fig. 3c). We also found that older adults experienced 620 positive emotions for LE more than HE videos (see Fig. 3a). Moreover, despite a restricted age 621 range in our sample consisting solely of elderly participants (from 65 to 83 years old), we observed 622 that the older the participants, the lower the negative and the higher the positive emotions reported 623 to exposure of others' suffering (see Fig. 3c), consistent with the "positivity bias" often reported in 624 the elderly ${ }^{7}$. This bias may reflect a motivation to upregulate positive and downregulate negative 625 information from external emotional stimuli ${ }^{7}$. Here, we additionally found that this pattern was modulated by levels of empathy: higher empathy correlated not only with increased negative affect 627 during HE videos, but also with increased positive affect during LE (see Fig. 3b). These results 628 indicate that empathy is associated with positive emotions in older adults when confronted with 629 social stimuli without overt emotional content, and offer a new perspective on the potential role of 630 empathy in the "positivity bias" of older adults. 


\section{Brain activity markers of empathy in older adults}

633 Brain responses to others' suffering (contrast $\mathrm{HE}>\mathrm{LE}$ videos) implicated several regions 634 overlapping with networks previously associated with social cognition and emotion. These 635 encompassed regions related to the affective empathy network, pain processing, or more generally 636 salience detection (aMCC, AI), as well as regions of the theory of mind (ToM) network (PCC, rTPJ, $637 \mathrm{dMPFC}$ ), and parts of the compassion network (ventral striatum) ${ }^{64,67}$. These results converge with 638 abundant work on neural activity in aMCC and AI related to empathy for pain ${ }^{35,44}$, encoding 639 behaviorally salient information ${ }^{72,73}$ and negative affect ${ }^{74,75}$. Brain regions such as TPJ and dMPFC 640 are engaged in scenarios requiring cognitive abilities to infer other's affective and mental state ${ }^{76,77}$, 641 and therefore related to cognitive aspects of empathy and theory of mind ${ }^{35,66}$. Interestingly, the

642 HE $>$ LE contrast also activated a cluster in ventral striatum, an area often associated with positive 643 affect and reward ${ }^{78}$ and engaged during compassion for other's suffering ${ }^{56,64}$.

644 On the other hand, there was no significant activation in the amygdala during the HE $>$ LE 645 videos, despite its well-known role in processing emotional stimuli. This null result might accord 646 with the notion that the amygdala responds more broadly to social or self-relevant information rather 647 than just negative valence ${ }^{79,80}$, and hence already activated to the content of LE videos. This would 648 be consistent with similar increases seen during both video conditions in our study (Supplementary 649 Fig. 1 and Fig. 5f).

650 Overall, our results suggest that socio-affective functions and brain regions mediating 651 empathy and theory of mind exhibit globally normal patterns of engagement in response to complex 652 negative social situations in healthy elderly. These data also demonstrate that our video paradigm 653 effectively engaged emotion and empathy processes in our participants, and that healthy elderly 654 individuals show evidence of positive affective biases in both behavioral and neural responses to 655 social scenes, indicating preserved empathy and emotional balance in this group.

\section{Emotional inertia and recovery from emotions after exposure to others' suffering}

658 Beyond transient responses to negative stimuli, assessing the impact of emotions over time is crucial to determine how people cope with stressful events ${ }^{81}$. Emotional inertia denotes a persistence of 660 emotional states ${ }^{16}$ indicating inefficient recovery and greater risk for psychological maladjustment ${ }^{22,81-83}$. Although well-studied behaviorally ${ }^{16,20}$, emotional inertia remains largely unexplored at the

662 brain level, especially in old populations. To uncover its neural underpinnings, we probed for carryover effects in brain activity at rest following exposure to emotional videos. 
666 DMN typically active at rest ${ }^{60}$, together with increases in amygdala and insula, two regions implicated in emotional processing ${ }^{36}$. The DMN is implicated in self-related internally-oriented processes including memory, interoception, and value-based decision making ${ }^{32,33}$. Interestingly, previous research found that the duration of activation in midline DMN regions was a better predictor of subjective emotional intensity of negative stimuli than the magnitude of activation ${ }^{26}$. A few other fMRI studies reported modulations of DMN in response to emotional challenges, although with divergent findings. While some researchers reported attenuated DMN activation following various emotions ${ }^{24,25}$, others reported increases ${ }^{26,31}$, similar to the current results. In the present study, we found both midline clusters of DMN (i.e., Precuneus/PCC and dMPFC) were not only activated in the HE > LE contrast during videos, but also continued their activity in the corresponding contrast during subsequent rest (post HE > post LE), providing direct evidence for "emotional inertia" in the brain.

Regarding other limbic regions, we observed that activity in the anterior insula was increased during both the (HE > LE) videos and the (post HE > post LE) rest periods, although the voxelwise activations did not fully overlap between the two conditions: while there was a more dorsal engagement during videos, more ventral parts of the anterior insula were active after the emotional event. In light of previous research in young adults ${ }^{84}$ suggesting that dorsal AI may be recruited during adaptive behavior mechanisms while ventral AI may be highly recruited during internal homeostatic regulation, our result may reflect a shift from controlled behavioral adaptation to more spontaneous homeostatic regulation. On the other hand, although the amygdala did not differentially respond during the $(\mathrm{HE}>\mathrm{LE})$ videos, it showed a lower return to baseline levels during rest after HE vs LE videos. Accordingly, previous research showed that prolonged amygdala activity after negative images predicted greater trait neuroticism ${ }^{37}$, and enhanced amygdala response to threat faces after negative emotion elicitation was amplified in high anxiety individuals ${ }^{19}$.

In line with these results, our data highlight the importance of the temporal dynamics of brain responses to emotion in order to determine individual affective styles and risks for psychopathology $24,26,29$.

693

\section{Brain connectivity patterns related to emotional inertia}

695 In addition, our functional connectivity analyses revealed that carryover effects in the brain were 696 organized in two dissociable circuits, linking the posterior core of DMN in PCC with right amygdala 697 and the anterior core in aMPFC with left anterior insula, whose respective coupling was selectively enhanced in the post HE relative to post LE rest condition (see Fig. 6). These results unveil a 
"fragmentation" of the midline DMN activity and its connectivity with limbic networks induced by emotional inertia, which was accompanied by distinctive behavioral features.

The PCC and amygdala were not only more active but also functionally more connected in the post-emotional rest periods, and the strength of this enhanced connectivity was predicted by individual anxiety and rumination. Thus, participants reporting higher rumination tendencies and anxious traits on questionnaires also exhibited stronger PCC-amygdala connectivity after HE videos. Explicit verbal reports also revealed that participants expressed more negative content in the thought probe pertaining to the rest period after HE videos, and participants with more frequent negative thoughts had higher PCC-amygdala connectivity compared to those who reported no negative thoughts. This was not the case after LE videos. These findings suggest that increased functional connectivity between PCC and amygdala may directly underpin the persistence of negative content in spontaneous thoughts. Past neuroimaging research suggests that PCC is involved in internally directed cognition and memory ${ }^{33}$ especially when people retrieve contextual and affective autobiographical information ${ }^{85,86}$. As the amygdala also plays a central role in affective memory by encoding and storing information about emotional relevance ${ }^{34,79,87}$, we speculate that PCC-amygdala communication may contribute to emotional inertia and recovery from socio-emotional stressful situations, potentially by associating the content of vicarious negative experiences to personal affective memories, especially in individuals with higher levels of anxiety and rumination. These data provide new insights on neural processes associated with rumination and repetitive negative thinking, i.e., mental states implying persistent self-relevant thoughts about negative information ${ }^{88}$ that are associated not only with maladaptive emotion regulation but also with increased risk of cognitive decline and $\mathrm{AD}{ }^{12,14}$. As neurodegenerative anomalies in PCC and medial brain regions are commonly seen in AD, changes in PCC connectivity might constitute a possible neural marker for deficient affective resilience, which is in turn associated with a higher risk for dementia.

In parallel, increased functional connectivity was also observed between AI and aMPFC after HE compared to LE videos. These neural changes showed no correlation with anxiety or rumination, but only a weak positive correlation with the empathic concern IRI subscale (see Supplementary Fig. 4). These findings may reflect a more general role of AI in emotional awareness ${ }^{89,90}$ and empathy ${ }^{35}$, and of aMPFC in the representation of affective states in both the self and others ${ }^{65,91}$. These results extend prior work by showing that modulation of connectivity between these two regions may occur not only during the appraisal of socio-emotional stimuli but also persist beyond emotional events. 


\section{Limitations and future directions}

734 Some limitations of the present study need to be acknowledged. First, while our findings extend previous work on empathy and emotional inertia that focused on young adults ${ }^{24,35}$, we did not include a young control group for comparison. Therefore, our data do not allow us to examine any age-dependent differences. However, the current study neatly dovetails with previous findings in the younger ${ }^{48,56}$, and provides a valuable cornerstone for future aging research. Second, we explicitly instructed our participants to watch the videos passively, and therefore some of the subsequent carryover effects on brain activity and connectivity could be interpreted as unsuccessful implicit emotion regulation styles inherent to the participants. It would be interesting to assess in future studies whether instructing participants with explicit emotion regulation strategies may change the subsequent brain response related to emotional inertia. Finally, we also acknowledge limitations related to the technical constraints of the fMRI scanner. As described in Supplementary Fig. 5, some basal forebrain voxels were automatically excluded from our group analyses due to magnetic field inhomogeneities frequently induced in brain regions near air-filled cavities in the human head ${ }^{92}$. Consequently, we were not able to study regions such as the orbitofrontal cortex (OFC), which plays an essential role in positive emotions and reward ${ }^{56,78}$.

\section{Conclusion}

751

752
In conclusion, the present study demonstrates that feelings of empathy for suffering and affective resilience can reliably be investigated in the elderly using the SoVT-Rest, a novel paradigm that has very low cognitive load and high ecological validity for future applications in frail or clinical populations. Using the SoVT-Rest, we find neural and behavioral markers of the positivity bias in the elderly and show for the first time lasting carryover effects (or emotional inertia) in corticolimbic brain circuits in a population of healthy older adults. Interestingly, increased functional connectivity of PCC and amygdala during rest after high emotional events was related to anxiety, rumination, and negative thought content, making this connectivity at rest a highly likely neural substrate for emotional inertia. These findings provide an important cornerstone for better understanding empathy and emotion regulation as well as their neural signatures in the aging population and thus contribute to identifying potential risk markers for neurodegenerative diseases associated with poor stress regulation. 


\section{Data Availability}

Data supporting the findings of the present manuscript are available upon request. To access to our data, please visit: https://silversantestudy.eu/2020/09/25/data-sharing/

\section{Code availability}

The code used to produce the results reported in the manuscript can be made available upon appropriate request.

\section{References}

1. Glisky, E. Changes in Cognitive Function in Human Aging. 3-20 (2007). doi:10.1201/9781420005523.sec1

2. Carstensen, L. L., Mayr, U., Pasupathi, M. \& Nesselroade, J. R. Emotional experience in everyday life across the adult life span. J. Pers. Soc. Psychol. 79, 644-655 (2000).

3. Mather, M. The Affective Neuroscience of Aging. (2015). doi:10.1146/annurev-psych122414-033540

4. Reiter, A. M. F., Kanske, P., Eppinger, B. \& Li, S.-C. The Aging of the Social Mind Differential Effects on Components of Social Understanding. Sci. Rep. 7, 11046 (2017).

5. Urry, H. L. \& Gross, J. J. Emotion regulation in older age. Curr. Dir. Psychol. Sci. 19, 352357 (2010).

6. Lang, F. R. \& Carstensen, L. L. Time counts: Future time perspective, goals, and social relationships. Psychol. Aging 17, 125-139 (2002).

7. Mather, M. \& Carstensen, L. L. Aging and motivated cognition: The positivity effect in attention and memory. Trends in Cognitive Sciences 9, 496-502 (2005).

8. Aldao, A., Nolen-Hoeksema, S. \& Schweizer, S. Emotion-regulation strategies across psychopathology: A meta-analytic review. Clin. Psychol. Rev. 30, 217-237 (2010).

9. Hamilton, J. P., Farmer, M., Fogelman, P. \& Gotlib, I. H. Depressive Rumination, the Default-Mode Network, and the Dark Matter of Clinical Neuroscience. Biol. Psychiatry 78, 224-230 (2015).

10. Kraaij, V., Pruymboom, E. \& Garnefski, N. Cognitive coping and depressive symptoms in the elderly: A longitudinal study. Aging Ment. Heal. 6, 275-281 (2002).

11. Terracciano, A. et al. Personality and risk of Alzheimer's disease: New data and metaanalysis. Alzheimer's Dement. 10, 179-186 (2014).

12. Marchant, N. L. \& Howard, R. J. Cognitive debt and Alzheimer's disease. J. Alzheimer's Dis. 44, 755-770 (2015).

13. Wilson, R. S., Begeny, C. T., Boyle, P. A., Schneider, J. A. \& Bennett, D. A. Vulnerability to Stress, Anxiety, and Development of Dementia in Old Age. Am. J. Geriatr. Psychiatry 19, 327-334 (2011).

14. Marchant, N. L. et al. Repetitive negative thinking is associated with amyloid, tau, and cognitive decline. Alzheimer's Dement. 1-11 (2020). doi:10.1002/alz.12116

15. Jané-Llopis, E. et al. Mental Health in Older People. (2008).

16. Kuppens, P., Allen, N. B. \& Sheeber, L. B. Emotional inertia and psychological maladjustment. Psychol. Sci. 21, 984-991 (2010). 
17. Koval, P., Kuppens, P., Allen, N. B. \& Sheeber, L. Getting stuck in depression: The roles of rumination and emotional inertia. Cogn. Emot. 26, 1412-1427 (2012).

18. Van De Leemput, I. A. et al. Critical slowing down as early warning for the onset and termination of depression. Proc. Natl. Acad. Sci. U. S. A. 111, 87-92 (2014).

19. Pichon, S., Miendlarzewska, E. A., Eryilmaz, H. \& Vuilleumier, P. Cumulative activation during positive and negative events and state anxiety predicts subsequent inertia of amygdala reactivity. Soc. Cogn. Affect. Neurosci. 10, 180-190 (2015).

20. Suls, J., Green, P. \& Hillis, S. Emotional reactivity to everyday problems, affective inertia, and neuroticism. Personal. Soc. Psychol. Bull. (1998). doi:10.1177/0146167298242002

21. Koval, P., Pe, M. L., Meers, K. \& Kuppens, P. Affect dynamics in relation to depressive symptoms: Variable, unstable or inert? Emotion 13, 1132-1141 (2013).

22. Trull, T. J., Lane, S. P., Koval, P. \& Ebner-Priemer, U. W. Affective Dynamics in Psychopathology. Emot. Rev. 7, 355-361 (2015).

23. Lamke, J. P. et al. The impact of stimulus valence and emotion regulation on sustained brain activation: Task-rest switching in emotion. PLoS One 9, (2014).

24. Eryilmaz, H., Van De Ville, D., Schwartz, S. \& Vuilleumier, P. Impact of transient emotions on functional connectivity during subsequent resting state: A wavelet correlation approach. Neuroimage 54, 2481-2491 (2011).

25. Pitroda, S., Angstadt, M., McCloskey, M. S., Coccaro, E. F. \& Phan, K. L. Emotional experience modulates brain activity during fixation periods between tasks. Neurosci. Lett. 443, 72-76 (2008).

26. Waugh, C. E., Hamilton, J. P. \& Gotlib, I. H. The neural temporal dynamics of the intensity of emotional experience. Neuroimage 49, 1699-1707 (2010).

27. Veer, I. M. et al. Beyond acute social stress: Increased functional connectivity between amygdala and cortical midline structures. Neuroimage 57, 1534-1541 (2011).

28. Eryilmaz, H., Van De Ville, D., Schwartz, S. \& Vuilleumier, P. Lasting impact of regret and gratification on resting brain activity and its relation to depressive traits. J. Neurosci. 34, 7825-7835 (2014).

29. Waugh, C. E., Hamilton, J. P., Chen, M. C., Joormann, J. \& Gotlib, I. H. Neural temporal dynamics of stress in comorbid major depressive disorder and social anxiety disorder. Biol. Mood Anxiety Disord. 2, 1-15 (2012).

30. Northoff, G., Qin, P. \& Nakao, T. Rest-stimulus interaction in the brain: A review. Trends Neurosci. 33, 277-284 (2010).

31. Schneider, F. et al. The resting brain and our self: Self-relatedness modulates resting state neural activity in cortical midline structures. Neuroscience 157, 120-131 (2008).

32. Raichle, M. E. et al. A default mode of brain function. Proc. Natl. Acad. Sci. (2001). doi:10.1073/pnas.98.2.676

33. Buckner, R. L., Andrews-Hanna, J. R. \& Schacter, D. L. The brain's default network: Anatomy, function, and relevance to disease. Ann. N. Y. Acad. Sci. 1124, 1-38 (2008).

34. LeDoux, J. The emotional brain, fear, and the amygdala. Cellular and Molecular Neurobiology (2003). doi:10.1023/A:1025048802629

35. Lamm, C., Decety, J. \& Singer, T. Meta-analytic evidence for common and distinct neural networks associated with directly experienced pain and empathy for pain. Neuroimage $\mathbf{5 4}$, 2492-2502 (2011).

36. Meaux, E. \& Vuilleumier, P. Emotion Perception and Elicitation. Brain Mapping: An Encyclopedic Reference 3, (Elsevier Inc., 2015).

37. Schuyler, B. S. et al. Temporal dynamics of emotional responding: Amygdala recovery predicts emotional traits. Soc. Cogn. Affect. Neurosci. 9, 176-181 (2014).

38. Kim, M. J. et al. The structural and functional connectivity of the amygdala: From normal emotion to pathological anxiety. Behav. Brain Res. 223, 403-410 (2011). 
39. Rey, G. et al. Resting-state functional connectivity of emotion regulation networks in euthymic and non-euthymic bipolar disorder patients. Eur. Psychiatry 34, 56-63 (2016).

40. Gross, J. J. The emerging field of emotion regulation: An integrative review. Review of General Psychology (1998). doi:10.1037/1089-2680.2.3.271

41. Chen, Y. C., Chen, C. C., Decety, J. \& Cheng, Y. Aging is associated with changes in the neural circuits underlying empathy. Neurobiol. Aging 35, 827-836 (2014).

42. Hühnel, I., Fölster, M., Werheid, K. \& Hess, U. Empathic reactions of younger and older adults: No age related decline in affective responding. J. Exp. Soc. Psychol. 50, 136-143 (2014).

43. Moore, R. C., Dev, S. I., Jeste, D. V., Dziobek, I. \& Eyler, L. T. Distinct neural correlates of emotional and cognitive empathy in older adults. Psychiatry Res. - Neuroimaging 232, 42-50 (2015).

44. Fan, Y., Duncan, N. W., de Greck, M. \& Northoff, G. Is there a core neural network in empathy? An fMRI based quantitative meta-analysis. Neurosci. Biobehav. Rev. 35, 903911 (2011).

45. Krueger, K. R. et al. Social engagement and cognitive function in old age. Exp. Aging Res. 35, 45-60 (2009).

46. MacLeod, S., Musich, S., Hawkins, K., Alsgaard, K. \& Wicker, E. R. The impact of resilience among older adults. Geriatr. Nurs. (Minneap). 37, 266-272 (2016).

47. Zunzunegui, M. V., Alvarado, B. E., Del Ser, T. \& Otero, A. Social networks, social integration, and social engagement determine cognitive decline in community-dwelling Spanish older adults. Journals Gerontol. - Ser. B Psychol. Sci. Soc. Sci. (2003). doi:10.1093/geronb/58.2.S93

48. Klimecki, O. M., Leiberg, S., Lamm, C. \& Singer, T. Functional neural plasticity and associated changes in positive affect after compassion training. Cereb. Cortex 23, 15521561 (2013).

49. Poisnel, G. et al. The Age-Well randomized controlled trial of the Medit-Ageing European project: Effect of meditation or foreign language training on brain and mental health in older adults. Alzheimer's Dement. Transl. Res. Clin. Interv. 4, 714-723 (2018).

50. Faul, F., Erdfelder, E., Lang, A.-G. \& Buchner, A. G*Power 3: A flexible statistical power analysis program for the social, behavioral, and biomedical sciences. Behav. Res. Methods 39, 175-191 (2007).

51. Davis, M. H. \& Association, A. P. A multidimensional approach to individual differences in empathy. JSAS Cat. Sel. Doc. Psychol. (1980).

52. Sheikh, J. I. \& Yesavage, J. A. 9/geriatric depression scale (Gds) recent evidence and development of a shorter version. Clin. Gerontol. (1986). doi:10.1300/J018v05n01_09

53. Spielberger, C. D., Gorsuch, R. L. \& Lushene, R. State-trait anxiety inventory STĀI (Form Y). Redw. City Mind Gard. (1983). doi:10.1515/9783111677439-035

54. Gross, J. J. \& John, O. P. Individual differences in two emotion regulation processes: Implications for affect, relationships, and well-being. J. Pers. Soc. Psychol. 85, 348-362 (2003).

55. Treynore, Gonzalez \& Nolen-Hoeksema, S. Ruminative Responses Scale. Cognit. Ther. Res. (2003). doi:10.1017/CBO9781107415324.004

56. Klimecki, O. M., Leiberg, S., Ricard, M. \& Singer, T. Differential pattern of functional brain plasticity after compassion and empathy training. Soc. Cogn. Affect. Neurosci. 9, 873-879 (2013).

57. Benjamini, Y. \& Hochberg, Y. Controlling the False Discovery Rate: A Practical and Powerful Approach to Multiple Testing. J. R. Stat. Soc. Ser. B (1995). doi:10.1111/j.25176161.1995.tb02031.x

58. Villain, N. et al. A simple way to improve anatomical mapping of functional brain imaging. 
J. Neuroimaging 20, 324-333 (2010).

912

913

914

915

916

917

918

919

920

921

922

923

924

925

926

927

928

929

930

931

932

933

934

935

936

937

938

939

940

941

942

943

944

945

946

947

948

949

950

951

952

953

954

955

956

957

958

959

960

59. Power, J. D., Barnes, K. A., Snyder, A. Z., Schlaggar, B. L. \& Petersen, S. E. Spurious but systematic correlations in functional connectivity MRI networks arise from subject motion. Neuroimage 59, 2142-2154 (2012).

60. Andrews-Hanna, J. R., Reidler, J. S., Sepulcre, J., Poulin, R. \& Buckner, R. L. FunctionalAnatomic Fractionation of the Brain's Default Network. Neuron 65, 550-562 (2010).

61. Fair, D. A. et al. A method for using blocked and event-related fMRI data to study 'resting state' functional connectivity. Neuroimage 35, 396-405 (2007).

62. Friston, K. J. Functional and effective connectivity in neuroimaging: A synthesis. Hum. Brain Mapp. 2, 56-78 (1994).

63. Winkler, A. M., Ridgway, G. R., Webster, M. A., Smith, S. M. \& Nichols, T. E. Permutation inference for the general linear model. Neuroimage 92, 381-397 (2014).

64. Singer, T. \& Klimecki, O. M. Empathy and compassion. Curr. Biol. 24, R875-R878 (2014).

65. Corradi-Dell' Acqua, C., Hofstetter, C. \& Vuilleumier, P. Cognitive and affective theory of mind share the same local patterns of activity in posterior temporal but not medial prefrontal cortex. Soc. Cogn. Affect. Neurosci. 9, 1175-1184 (2014).

66. Schurz, M., Radua, J., Aichhorn, M., Richlan, F. \& Perner, J. Fractionating theory of mind: A meta-analysis of functional brain imaging studies. Neurosci. Biobehav. Rev. 42, 9-34 (2014).

67. Preckel, K., Kanske, P. \& Singer, T. On the interaction of social affect and cognition: empathy, compassion and theory of mind. Curr. Opin. Behav. Sci. 19, 1-6 (2018).

68. Epstein, R. \& Kanwisher, N. A cortical representation the local visual environment. Nature (1998). doi:10.1038/33402

69. Aguirre, G. K., Detre, J. A., Alsop, D. C. \& D'Esposito, M. The parahippocampus subserves topographical learning in man. Cereb. Cortex (1996). doi:10.1093/cercor/6.6.823

70. Boisgueheneuc, F. et al. Functions of the left superior frontal gyrus in humans : a lesion study. 3315-3328 (2006). doi:10.1093/brain/awl244

71. Klimecki, O. et al. The impact of meditation on healthy ageing — the current state of knowledge and a roadmap to future directions. Curr. Opin. Psychol. 28, 223-228 (2019).

72. Seeley, W. W. et al. Dissociable intrinsic connectivity networks for salience processing and executive control. J. Neurosci. 27, 2349-2356 (2007).

73. Menon, V. \& Uddin, L. Q. Saliency, switching, attention and control: a network model of insula function. Brain Struct. Funct. 214, 655-667 (2010).

74. Knutson, B., Katovich, K. \& Suri, G. Inferring affect from fMRI data. Trends Cogn. Sci. 18, 422-428 (2014).

75. Corradi-Dell'Acqua, C., Tusche, A., Vuilleumier, P. \& Singer, T. Cross-modal representations of first-hand and vicarious pain, disgust and fairness in insular and cingulate cortex. Nat. Commun. 7, (2016).

76. Bruneau, E. G., Jacoby, N. \& Saxe, R. Empathic control through coordinated interaction of amygdala, theory of mind and extended pain matrix brain regions. Neuroimage 114, 105119 (2015).

77. Bruneau, E., Dufour, N. \& Saxe, R. How We Know It Hurts: Item Analysis of Written Narratives Reveals Distinct Neural Responses to Others' Physical Pain and Emotional Suffering. PLoS One 8, (2013).

78. Knutson, B., Fong, G. W., Adams, C. M., Varner, J. L. \& Hommer, D. Dissociation of reward anticipation and outcome with event-related fMRI. Neuroreport 12, 3683-3687 (2001).

79. Sander, D., Grafman, J. \& Zalla, T. The Human Amygdala: An Evolved System for Relevance Detection. Reviews in the Neurosciences (2003). 
80. Cunningham, W. A., Raye, C. L. \& Johnson, M. K. 0898929042947919. 1-13 (2004).

81. Davidson, R. J. Affective Style and Affective Disorders: Perspectives from Affective Neuroscience. Cogn. Emot. 12, 307-330 (1998).

82. Davidson, R. J. Well-being and affective style: Neural substrates and biobehavioural correlates. Philos. Trans. R. Soc. B Biol. Sci. 359, 1395-1411 (2004).

83. Koval, P., Butler, E. A., Hollenstein, T., Lanteigne, D. \& Kuppens, P. Emotion regulation and the temporal dynamics of emotions: Effects of cognitive reappraisal and expressive suppression on emotional inertia. Cogn. Emot. 29, 831-851 (2015).

84. Lamm, C. \& Singer, T. The role of anterior insular cortex in social emotions. Brain Struct. Funct. 214, 579-591 (2010).

85. Addis, D. R., Wong, A. T. \& Schacter, D. L. Remembering the past and imagining the future: Common and distinct neural substrates during event construction and elaboration. Neuropsychologia 45, 1363-1377 (2007).

86. Mason, M. F. et al. Wandering minds: The default network and stimulus-independent thought. Science (80-. ). 315, 393-395 (2007).

87. Phelps, E. A. \& LeDoux, J. E. Contributions of the amygdala to emotion processing: From animal models to human behavior. Neuron 48, 175-187 (2005).

88. Ehring, T. \& Watkins, E. R. Repetitive Negative Thinking as a Transdiagnostic Process. Int. J. Cogn. Ther. 1, 192-205 (2008).

89. Critchley, H. D., Wiens, S., Rotshtein, P., Öhman, A. \& Dolan, R. J. Neural systems supporting interoceptive awareness. Nat. Neurosci. 7, 189-195 (2004).

90. Zaki, J., Davis, J. I. \& Ochsner, K. N. Overlapping activity in anterior insula during interoception and emotional experience. Neuroimage 62, 493-499 (2012).

91. Amodio, D. M. \& Frith, C. D. Meeting of minds : the medial frontal cortex and social cognition. 7, 268-277 (2006).

92. Juchem, C., Nixon, T. W., McIntyre, S., Rothman, D. L. \& De Graaf, R. A. Magnetic field homogenization of the human prefrontal cortex with a set of localized electrical coils. Magn. Reson. Med. 63, 171-180 (2010). 


\section{Acknowledgments}

994 The Age-Well randomized clinical trial is part of the Medit-Ageing project and is funded through

995 the European Union's Horizon 2020 Research and Innovation Program (grant 667696), Institut

996 National de la Santé et de la Recherche Médicale, Région Normandie, and Fondation d'Entreprise

997 MMA des Entrepreneurs du Futur. The authors are grateful to the Cyceron magnetic resonance

998 imaging staff for their help with recruitment and data acquisition and administrative support and to

999 all the participants of the study for their contribution. We acknowledge the members of the Medit-

1000 Ageing Research Group, and we thank Clara Bordas and Silvia de Cataldo for their help on the 1001 mental thoughts analyses. We are particularly grateful to Marc Heidmann for his support through 1002 different stages of this study.

1003

\section{Author contributions}

1005 Conceptualization: S.B.L., O.K., and P.V.; Data curation: S.B.L., Y.I.D.A., O.K., and P.V.; Formal 1006 analysis: S.B.L., and Y.I.D.A.; Funding acquisition: O.K., and P.V.; Investigation: S.B.L., and 1007 members of the Medit-Ageing Research Group; Methodology: S.B.L., O.K., and P.V.; Supervision: 1008 O.K., and P.V.; Visualization: S.B.L.; Writing - Original Draft: S.B.L.; Writing - Review and 1009 Editing: S.B.L., F.C., O.K., P.V., and Y.I.D.A.

\section{Competing interests}

1012

1013 The authors declare no competing interests. 


\section{Figures}

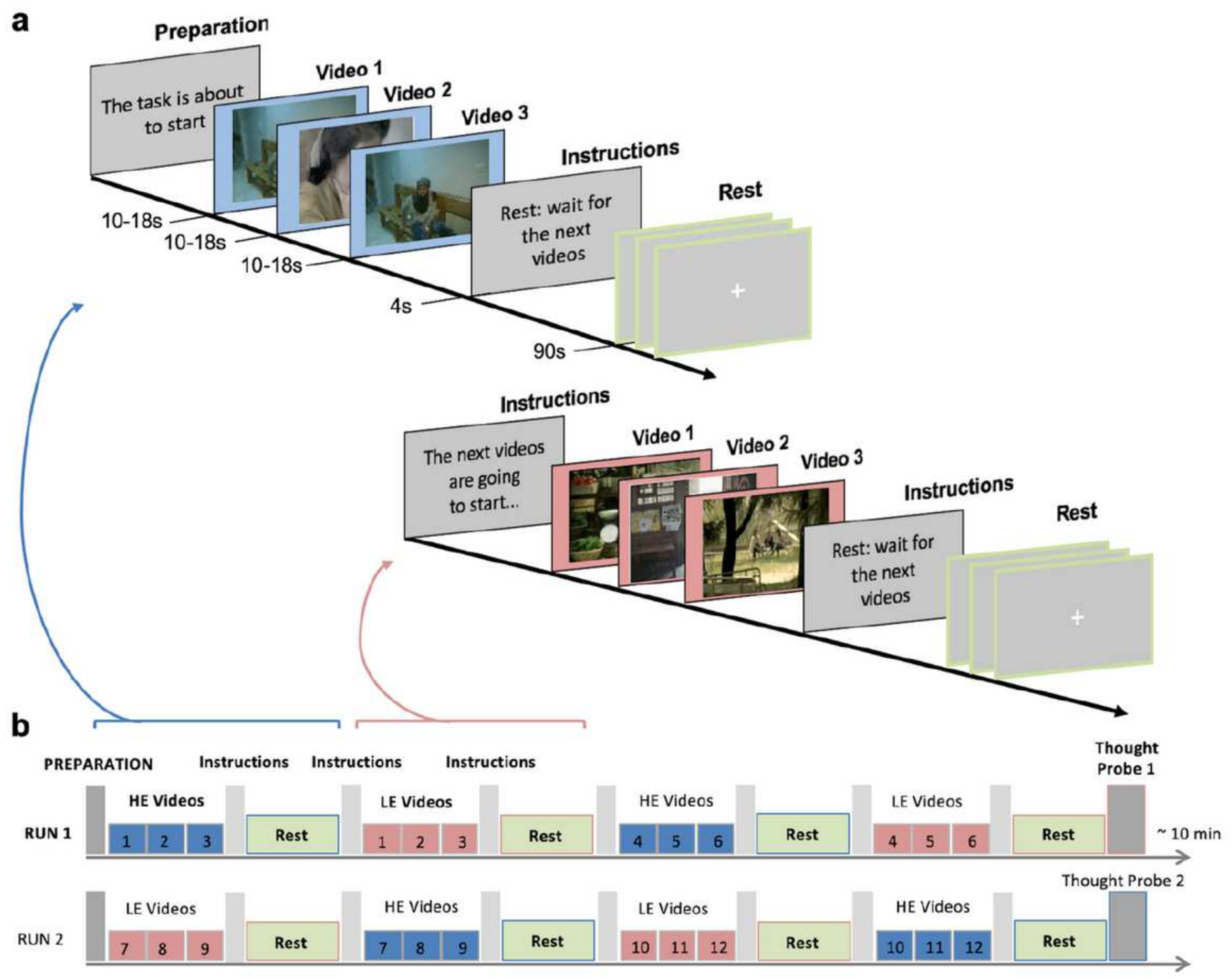

Figure 1

Experimental design: (a) SoVT-Rest paradigm: 12 High Emotion (HE) and 12 Low Emotion (LE) videos were presented grouped in blocks of three. HE videos depict suffering people (e.g., due to injuries or natural disasters), while LE videos depict people during everyday activities (e.g., walking or talking). Each block of three videos is followed by a resting state period of 90 seconds. (b) Each run ends with a thought probe in which participants verbally express what they had been thinking and/or feeling during the last rest period (via a microphone), once following a LE block and once following a HE block. The order of the runs was randomized between participants. 


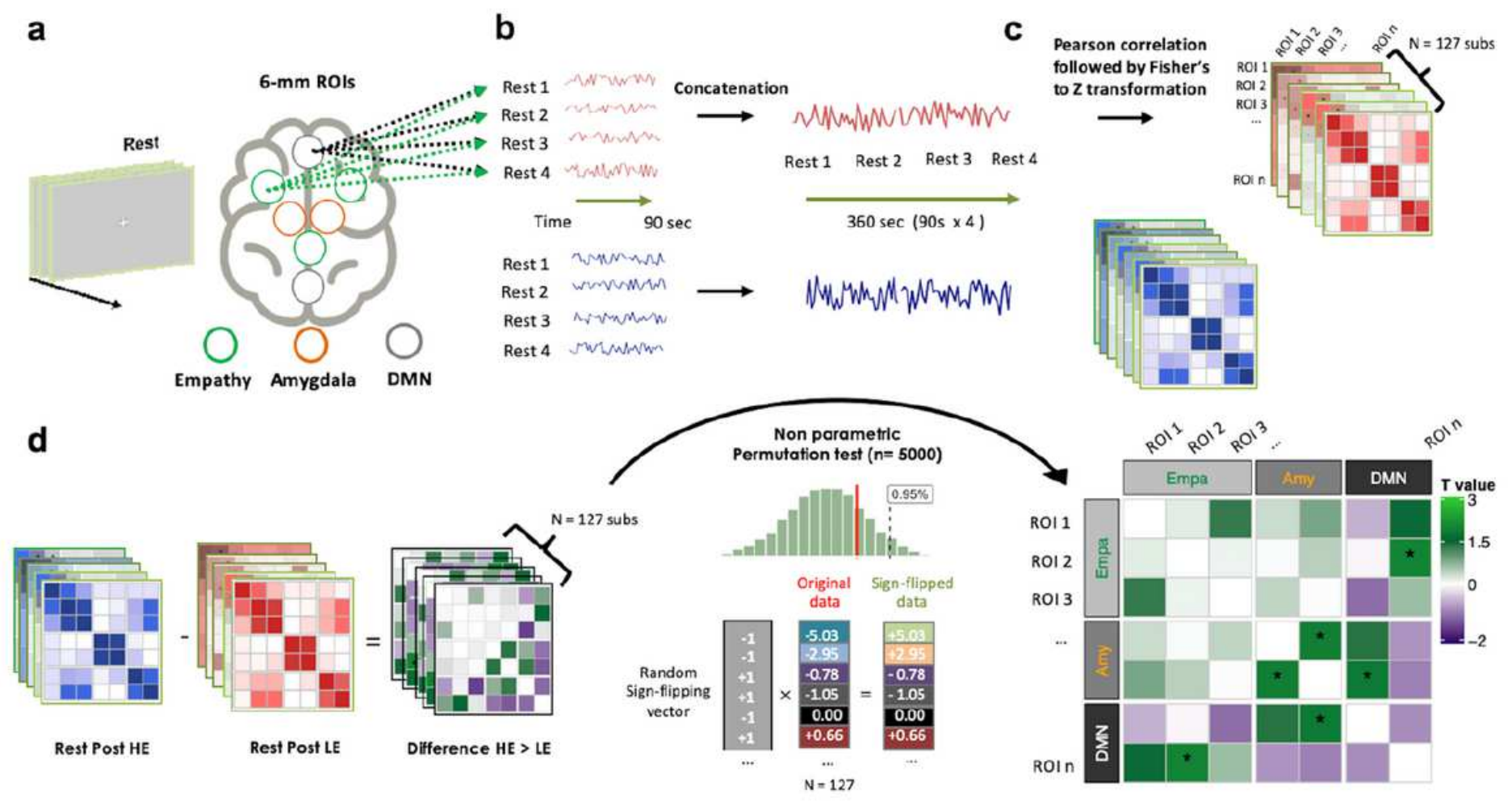

Figure 2

Functional connectivity pipeline: (a) Regions of interest (ROIs) from the default mode network (DMN) were chosen based on Andrews-Hanna et al. (2010), including the posterior cingulate cortex (PCC, - 8 -56 26) and anterior medial prefrontal cortex (aMPFC, -652 -2). ROls from the empathy network were based on the meta-analysis by Fan et al. (2011), including the bilateral anterior insula (Al, -36 162 and $3824-2$ ) and anterior mid cingulate cortex (aMCC, -2 24 38). A 6 mm-radius sphere was created for each ROI. The amygdala was defined anatomically using the SPM anatomical template. (b) For every participant, time series from the video and instruction periods were removed, and the remaining time series corresponding to the rest periods were concatenated 61 . The final concatenated time series of the four rest blocks for each type of video (high emotion, HE or low emotion, LE) resulted in 184 frames ( $₫ 360 \mathrm{~s}$ ) of resting-state data for each subject. (c) We then correlated the time-courses between the different ROIs using Pearson's $r$ correlation, and the resulting coefficients were Fisher's $r$ to $z$ transformed to improve normality in the data. Individual Z-score maps (correlation matrices) were created for each participant. (d) Finally, significant differences between the two correlation matrices (post HE rest vs. post LE rest) were tested using a nonparametric permutation test 63 . For each pair of nodes, the permutation test compared the true correlation difference treal (HE vs. LE) to a null distribution tpermuted constructed by randomly flipping the sign of the correlation coefficients and repeating the t statistic $(n=5000)$. 
a
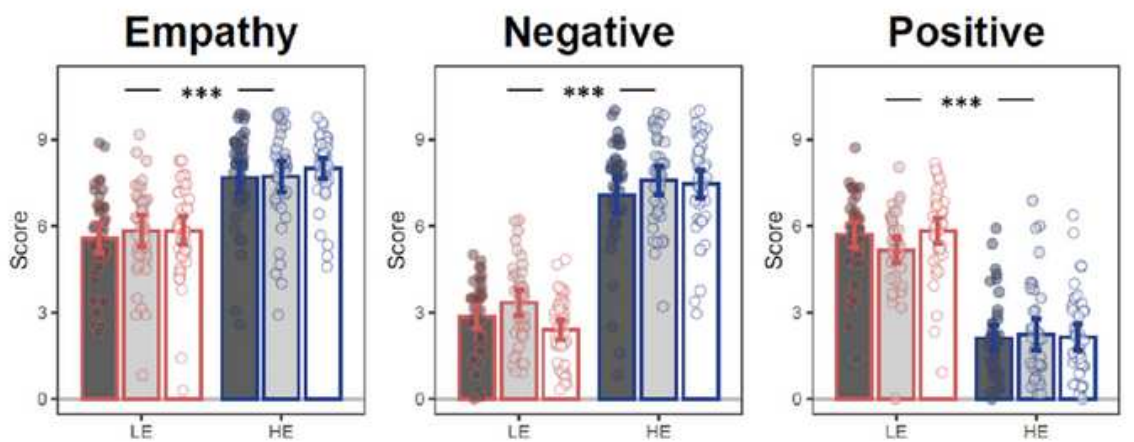

$* * *=p<0.001$

Videoset

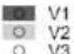

Video Type

Bigh Emotion

b
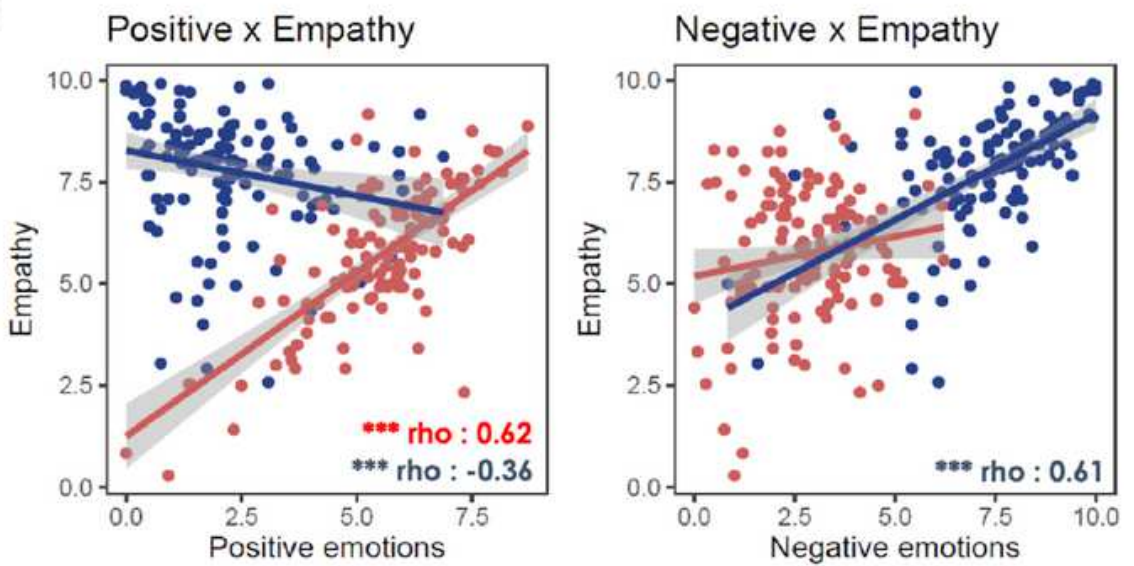

${ }^{* * *}=\mathrm{p}<0.001$

Video type

- Low Emotion

- High Emotion

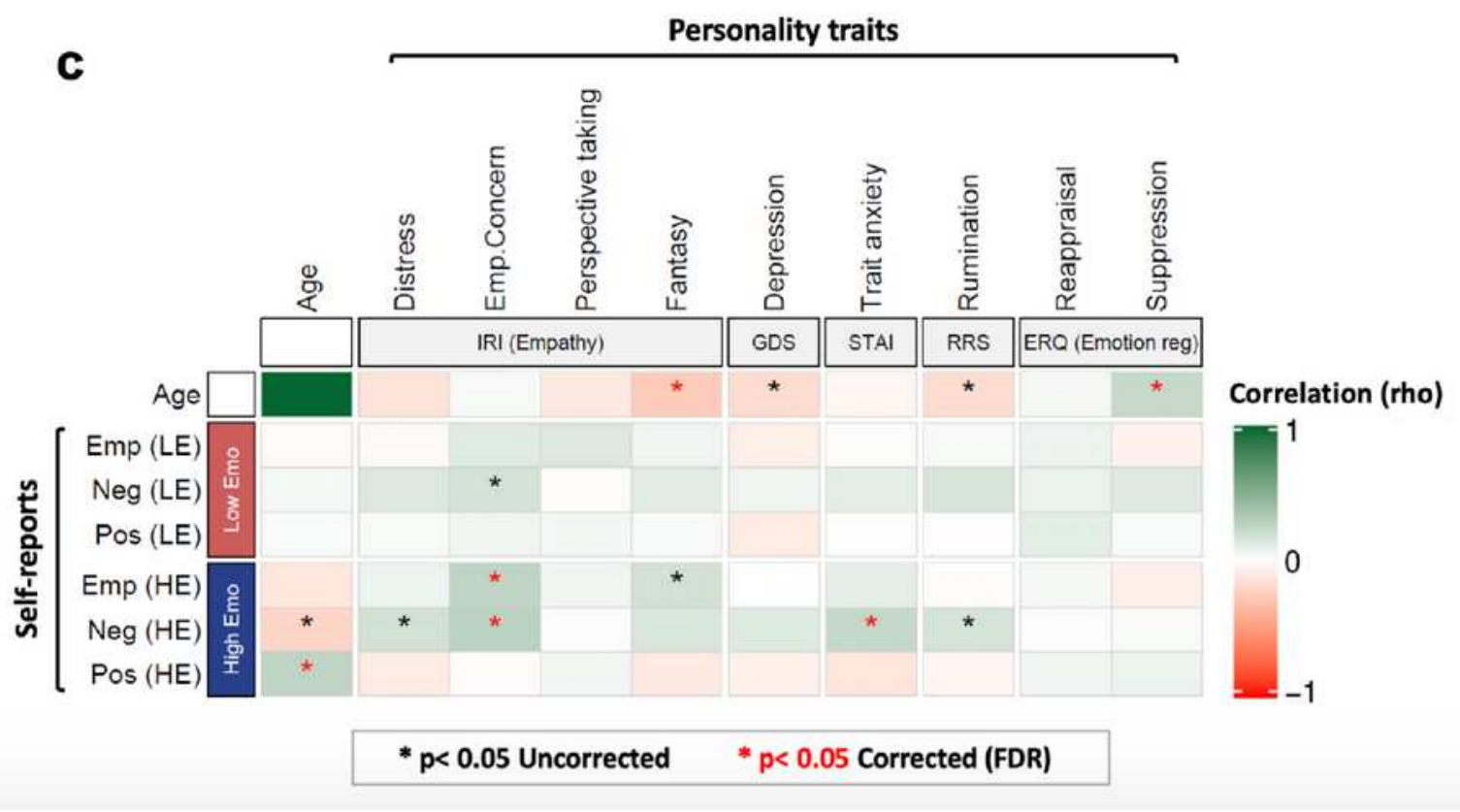

Figure 3

(a) Self-reported scores of empathy, positive affect, and negative affect for the HE and LE videos. (b) Spearman correlations between self-reported scores of empathy and affective ratings. Error bars represent $95 \%$ confidence intervals; dots represent averaged values for each participant per condition, $n=$ 127. (c) Spearman correlations between age, personality traits, and self-reported scores of empathy, positive affect, and negative affect. Blue: HE videos, Red: LE videos, $n=126$ (1 missing data point). IRI: 
Interpersonal Reactivity Index, GDS: Geriatric Depression Score, STAI: STAl-trait Anxiety Index, RRS: Rumination Response Scale, ERQ: Emotion Regulation Questionnaire.

\section{Videos : HE $>$ LE}

$(P<.05$ FWE-Corrected )

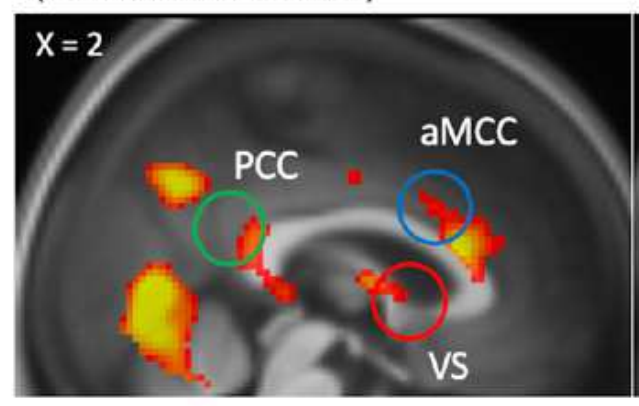

\subsection{T-value 9}

\section{$\mathrm{Z}=0$}

$X=-48$

$x=-12$

r. Al

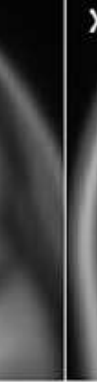

\section{Figure 4}

Brain regions with greater activation during high emotion (HE) videos in contrast to low emotion (LE) videos. Reported results are corrected for multiple comparisons using familywise error (FWE) correction at the voxel level ( $p<0.05$ FWE-corrected). Blue circles show regions previously reported as part of the Empathy network (bilateral anterior insula, Al; anterior middle cingulate cortex, aMCC), green circles show regions previously reported as part of the Theory of Mind network (PCC: posterior cingulate cortex, I. TPJ: left temporo-parietal junction, dMPFC: dorsal medial prefrontal cortex), red circles show regions associated with the Compassion network (VS: ventral striatum) 64,67. Activations are displayed on the average $\mathrm{T} 1$ image of our 127 participants. 

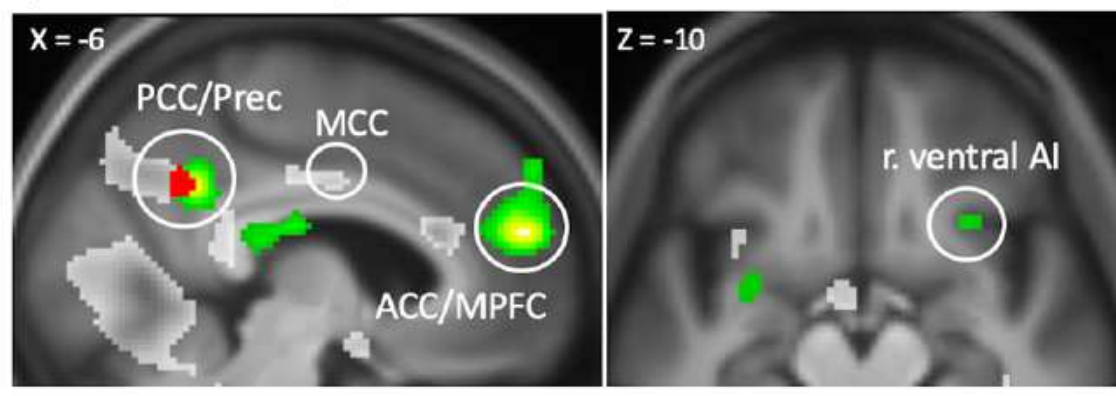

4.5 T-value 8

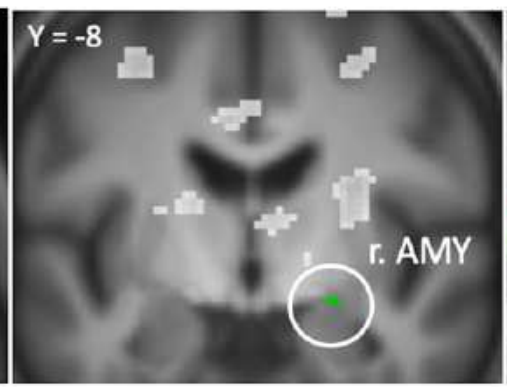

b

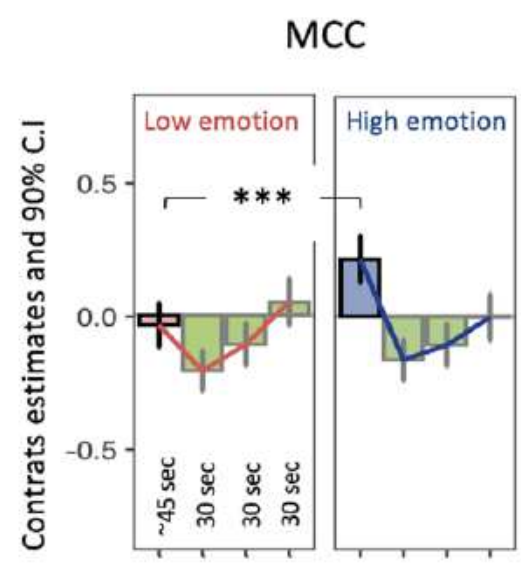

Time periods

e

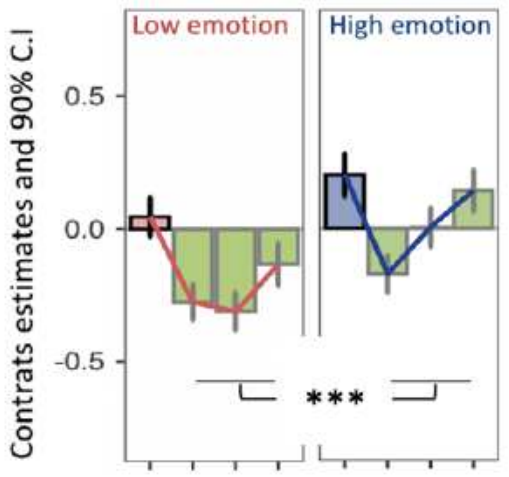

Time periods

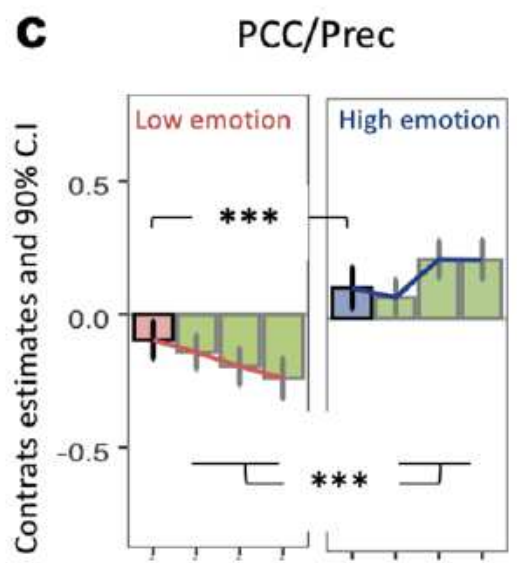

Time periods

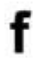

r. AMY

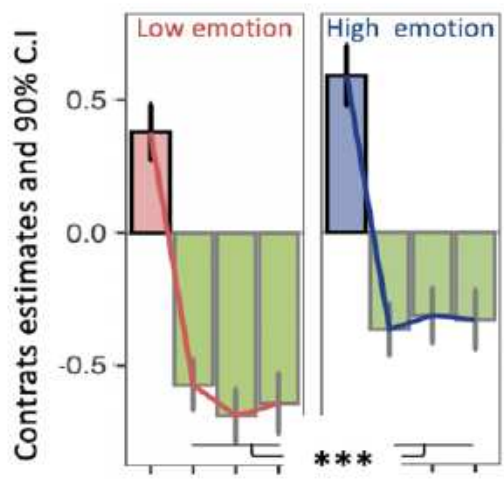

Time periods d $\quad$ dMPFC

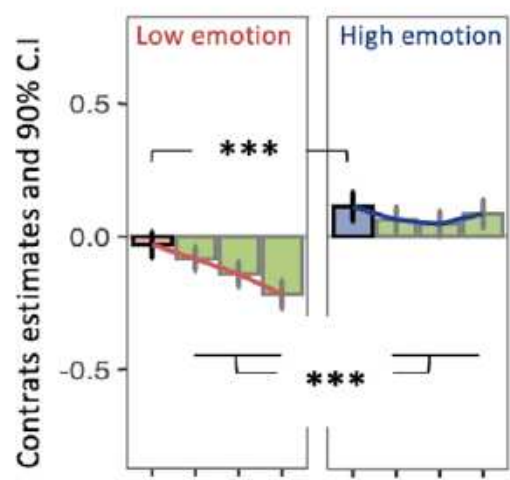

Time periods

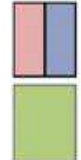

Videos periods

Rest periods

\section{Figure 5}

Brain activations to high emotion (HE) versus low emotion (LE) videos and corresponding carryover effects during rest periods. (a) Clusters in grey show brain regions significantly activated in the contrast $\mathrm{HE}$ videos > LE videos. Clusters in green show brain regions significantly activated in the rest periods corresponding to the contrast post HE videos > post LE videos. Red clusters show the overlap. Reported results are $p<0.05$ corrected for multiple comparisons using family-wise error (FWE) correction at the 
voxel level. (b,c,d,e,f) Magnitude and time-course of brain activity (parameter estimates) for relevant regions during the different task periods. (b) Example of a region (in MCC) responding to HE vs LE videos, but showing no significant difference in activation during rest after HE vs LE videos. (c,d) Example of regions (PCC/Prec and dMPFC) responding to HE > LE videos and showing significant carryover with sustained activity during subsequent rest. $(e, f)$ The right amygdala as well as the ventral part of the right anterior insula did not reliably respond to HE vs LE videos but showed significant increases in activations during corresponding rest. Pink lines track activity time-courses during LE conditions, blue lines track activity time-courses during HE conditions. Pink and blue bars indicate activity (blocks of 3 videos $=\sim 45$ seconds) for LE and HE videos respectively, green bars indicate activity (over 3 bins of 30 seconds) during rest periods subsequent to corresponding videos periods. Activations are displayed on the average T1 image of our 127 participants. ${ }^{\star \star \star} p<0.05$ FWE-corrected. PCC: posterior cingulate cortex, Prec: precuneus, MCC: midcingulate cortex, ACC: anterior cingulate cortex, MPFC: medial prefrontal cortex, dMPFC: dorsal medial prefrontal cortex, r. ventral Al: right anterior insula (ventral part), r. AMY: right amygdala. 

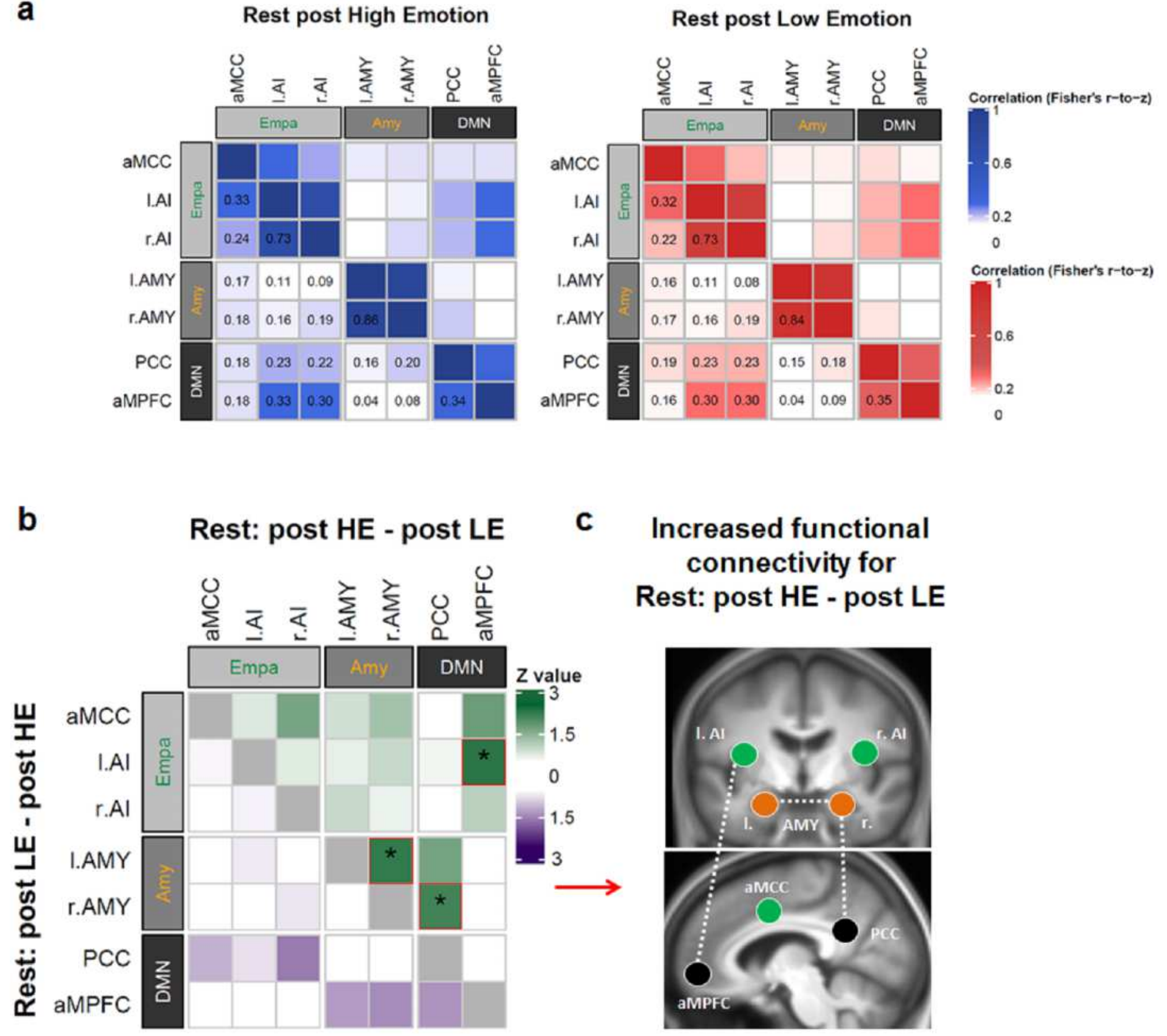

Figure 6

Functional connectivity results illustrated as (a) correlation matrices between pairs of ROls for the different rest conditions. Blue matrix corresponds to post HE (high emotion) rest periods, red to post LE (low emotion) rest periods. The plotted values (correlation coefficients following Fisher's r-to-z transformation) were obtained by averaging the $n=127$ correlation matrices for each condition. (b) Correlation matrix corresponding to the difference between the two rest conditions, showing post emotion increases (green) and post emotion decreases (violet). Left and right halves of the matrix with respect to the diagonal depict the values for inverse contrasts (upper part of the matrix: post HE - post LE rest periods; lower part of the matrix: post LE - post HE rest periods. Significant changes in correlations with Z $>1.64$ are marked by an asterisk * corresponding to $p<0.05$, one-tailed uncorrected). (c) Visual 
representations of significant changes in functional connections. Black ROls= DMN regions, Orange ROls = bilateral amygdala, Green ROIs = empathy network regions. Al: anterior insula, aMCC: anterior midcingulate cortex, AMY: amygdala, PCC: posterior cingulate cortex, aMPFC: anterior medial prefrontal cortex. The brain image corresponds to the average of the $127 \mathrm{~T} 1$ images of our sample.
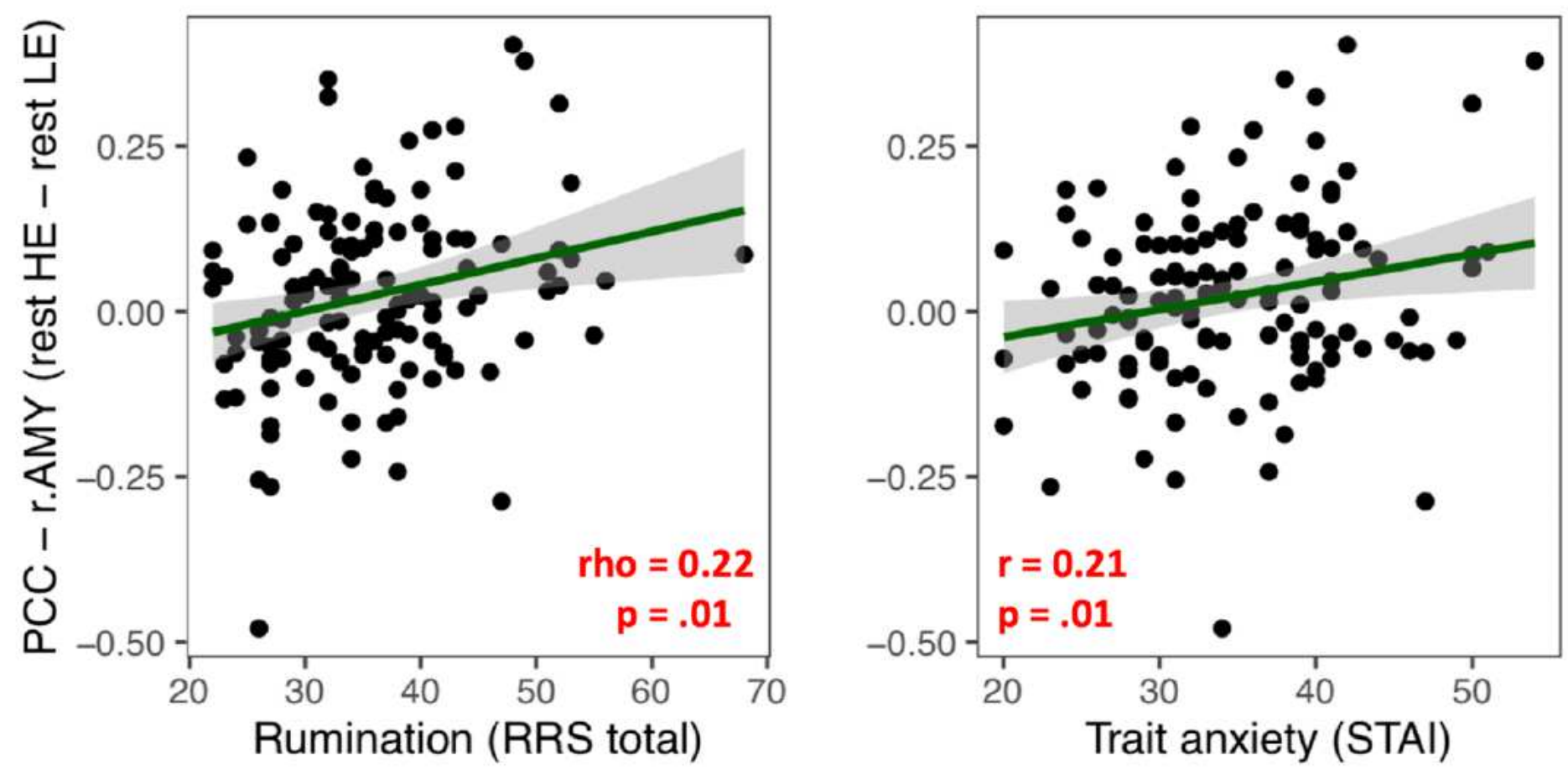

Figure 7

Pearson ( $r$ ) and Spearman (rho) correlations show that higher functional connectivity between posterior cingulate cortex and amygdala during rest periods after HE > LE videos [PCC-r.AMY(rest HE-rest LE)] was positively related to trait anxiety (STAI.B) and rumination (RRS total). 
a

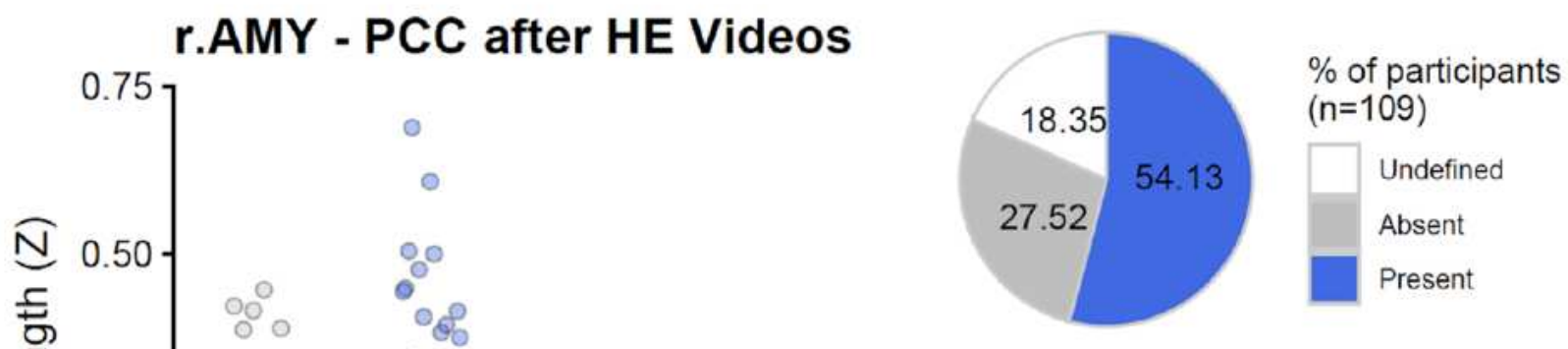

Negative

Thoughts

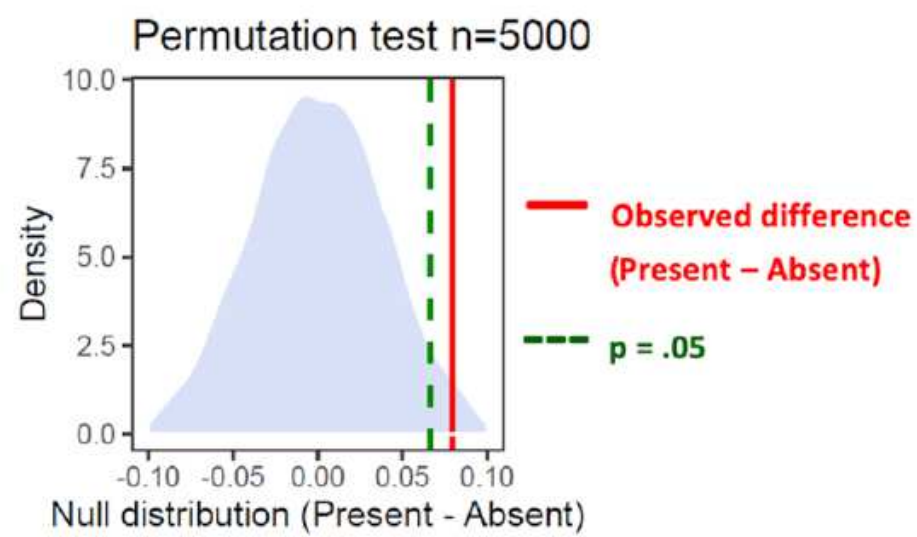

b
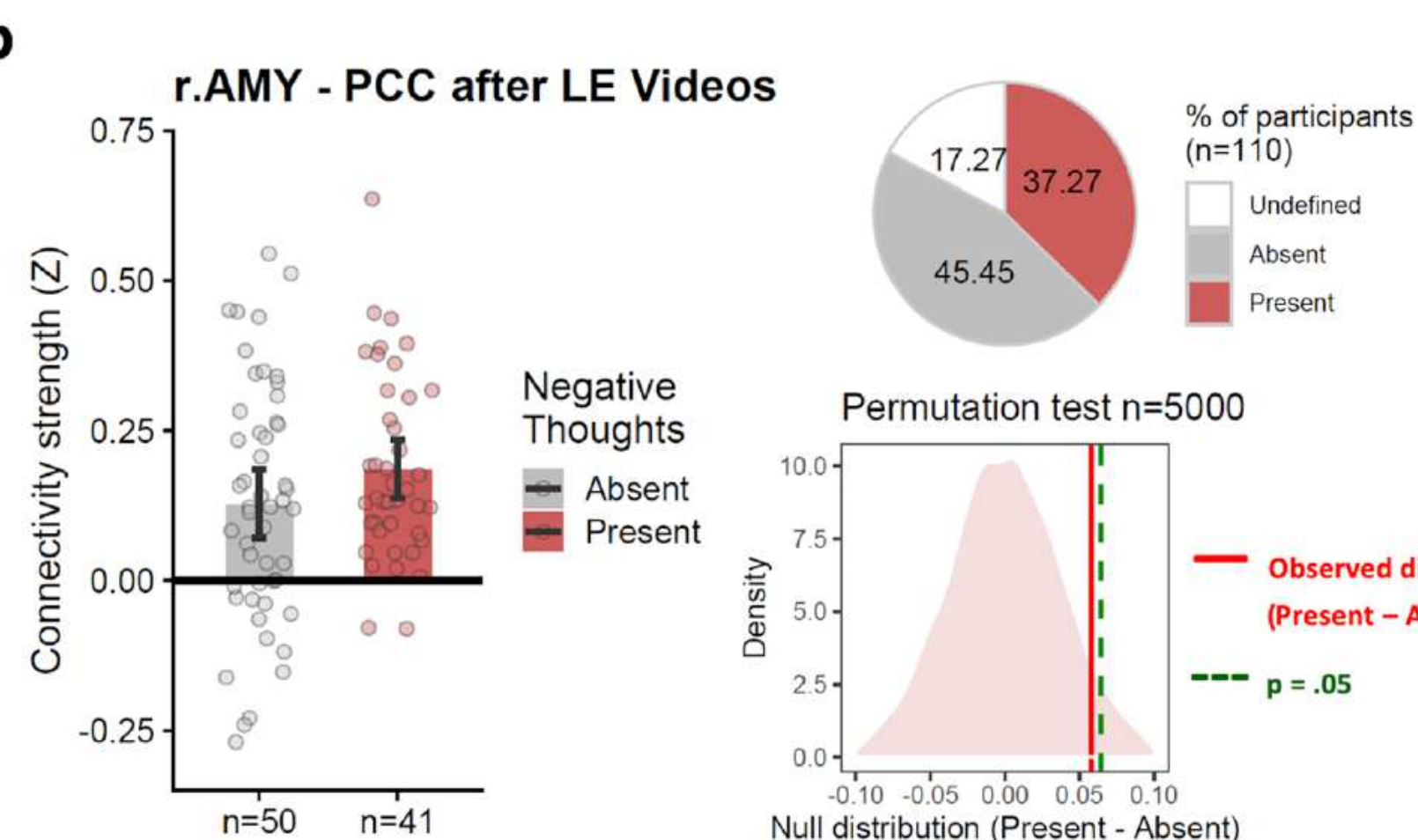

Absent

Present

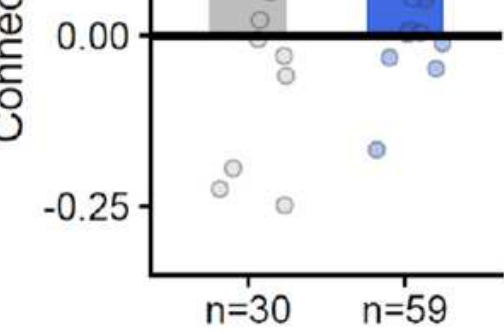

Negative

Absent

Present

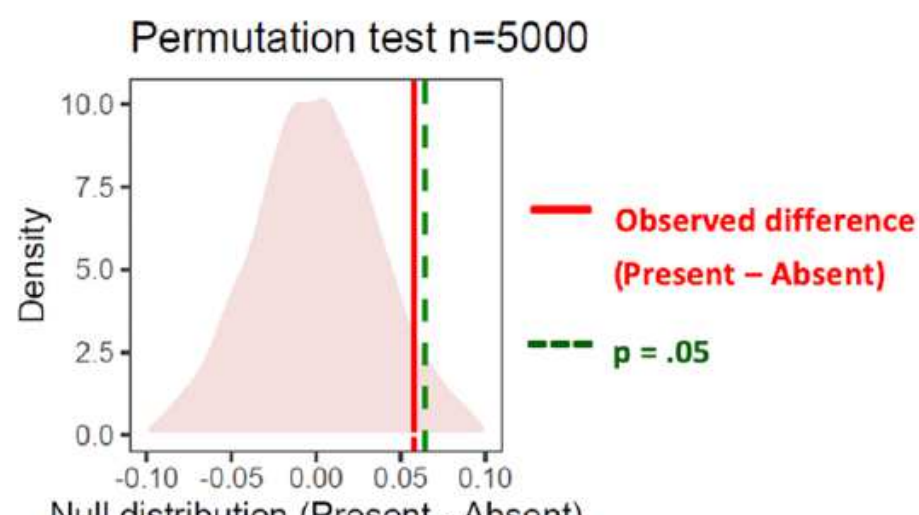

Null distribution (Present - Absent)

\section{Figure 8}

$(a, b)$ r.AMY-PCC connectivity between the group of participants that verbally reported negative content during the thought probes (Present) vs. the group that did not (Absent), for both HE and LE conditions. After HE videos, 59(54\%) participants reported negative content in their thought probes, 30(28\%) did not report negative content and 20(18\%) were ambiguous(undefined). After LE videos in turn, only $41(37 \%)$ reported negative content vs. 50(45)\% not reporting negative thoughts and $19(17 \%)$ were ambiguous 
(undefined). At the brain level, the comparison was made with a non-parametric permutation analysis in which the true mean PCC-AMY connectivity difference between the groups Present-Absent (observed diff $=0.08$ ) was compared to a null-distribution built by permuting the labels 5000 times. As hypothesized, we observed that $54 \%$ of the participants reporting negative content in their thoughts (vs. the $28 \%$ not reporting) showed increased PCC- r.AMY connectivity in the HE conditions ( $p=0.02$, one-tailed). In the LE conditions, there was no significant difference in PCC- r.AMY connection between the two groups (observed diff $=0.06 ; p=0.07$, one-tailed). Blue: High Emotion (HE) condition, Red: Low emotion (LE) condition. r.AMY-PCC: connectivity between the right amygdala and the posterior cingulate cortex. The percentages in the text are rounded.

\section{Supplementary Files}

This is a list of supplementary files associated with this preprint. Click to download.

- SupplMaterialBaezLugo.pdf

- MeditAgeingResearchGroupBaezLugo.pdf 